\title{
LA PRODUCTIVIDAD DE LA TIERRA \\ EN ESPAÑA 1752-1930: \\ TENDENCIA A LARGO PLAZO
}

\author{
MIGUEL ÁNGEL GUTIÉRREZ BRINGAS * \\ Universidad de Cantabria
}

\section{RESUMEN}

El objetivo fundamental de este trabajo es calcular la tendencia a largo plazo de la productividad de la tierra en España entre 1752 y 1930, utilizando para ello por primera vez una fuente prácticamente desconocida en la historiografia española, los Cuadernos Generales de la Riqueza (1818-1820).

Se llega a la conclusión de que a lo largo del siglo XIx las productividades agricolas crecieron en España de una forma considerable - un $70 \%$ para los cereales en las superficies sembradas y se duplican los rendimientos de los viñedos-, resultado que contradice la clásica opinión expresada por los historiadores que se han ocupado de este tema.

\section{ABSTRACT}

This paper seeks to calculate long term trends of land productivity in Spain, 17521930, using for it a practically unknown source in the Spanish historiography, the General Wealth Notebooks (1818-1820).

The conclusion of this study is that during the 19th century agricultural productivity grew substantially in Spain $-70 \%$ for the cereal in the sown land and doubled the yield in the vineyard-, contradicting the generally accepted view by historians.

El presente trabajo es un avance de la memoria de licenciatura que en la actualidad se está elaborando con el mismo titulo y que tiene como objetivo

* Esta investigación ha sido posible gracias a dos becas del Banco de España para los cursos 1989-1990 y 1990-1991. Quisiera además dejar constancia de mi agradecimiento a Sebastian Coll Martin, director de mi tesis doctoral, por su ayuda durante estos años. 
fundamental estudiar las superficies agrarias y las producciones agricolas, a fin de estar en condiciones de calcular la productividad de la tierra en España a lo largo de los siglos XvIII al xx. Esta investigación se enmarca dentro de un proyecto más amplio y ambicioso que persigue la estimación de la productividad total de los factores y la reconstrucción del producto agricola de nuestro pais durante la edad contemporánea.

Estas páginas nacen exclusivamente con el propósito de exponer las principales conclusiones sobre la tendencia a largo plazo de la productividad de la tierra - volumen de producción entre cantidad de superficie - en España para el periodo de 1752 a 1930, obtenidas a partir de la evidencia cuantitativa que proporcionan dos fuentes manuscritas de los años 1752 y 1818 (las Respuestas Generales del Catastro de Ensenada y los Cuadernos Generales de la Riqueza), así como las estadisticas publicadas a principios del siglo $\mathrm{xx}$.

El deficiente estado de nuestros conocimientos sobre este tema, a pesar de su importancia, al ser uno de los mejores indicadores económicos del progreso técnico en la agricultura, hace necesario que primero se conozca de una forma cuantificada y con unas bases lo más sólidas posibles su evolución -al menos en sus grandes líneas- y los niveles alcanzados por cada uno de los cultivos, para después poder analizar las causas del crecimiento de la producción agraria española del siglo XIX.

Desde el primer estudio cuantitativo sobre la historia de la productividad agraria en Europa de B. H. Slicher van Bath en 1963 ', que tuvo el mérito de impulsar numerosas investigaciones sobre este problema y de que fuera uno de los temas centrales de la III Conferencia Internacional de Historia Económica (Munich, 1965), hasta las publicaciones más recientes de Bairoch, Van Zanden o Campbell y Overton ${ }^{2}$ son numerosos los trabajos que se han ocupado de los rendimientos en la agricultura europea de los últimos siglos. No ocu. rre lo mismo en España, donde la bibliografía es escasa y no existe ninguna monografía específica, ya que el tema sólo ha sido tratado en algunos artículos o como un apartado dentro de capítulos sobre la economia agraria.

A finales de la década de los cincuenta, Vicens Vives planteó la primera visión sobre el desarrollo económico de España durante el siglo XIX, atribuyendo a la agricultura buena parte de la responsabilidad del atraso industrial al calificarla como un sector subdesarrollado y feudal, en el cual la productividad de la tierra se habria mantenido constante a lo largo del siglo; solamente habia

\footnotetext{
Con su trabajo «Yield ratios, 810-1820», en A.A.G. Bijdragen num. 10, año 1963.

Bairoch (1989), Van Zanden (1991), Campbell y Overton (1991).
} 
crecido la productividad del viñedo -en un 50 por 100-, basandose para ello en las controvertidas cifras de Salvador Millet

\begin{tabular}{|c|c|c|c|}
\hline & 1800 & 1860 & $1 \%(\%)$ \\
\hline Trigo & $6,3(100)$ & $5,8(92)$ & $6,9(109)$ \\
\hline Cereales & $6,4(100)$ & $6,2(96)$ & $7,0(109)$ \\
\hline$\ldots \ldots \ldots \ldots$ & $9,6(100)$ & $9,0(93)$ & $14,8(154)$ \\
\hline Olivar & - & $1,6(100)$ & $1,8(112)$ \\
\hline
\end{tabular}

Posteriormente, esta idea sobre la evolución de la productividad ha sido reforzada, más por suposiciones que en términos cuantitativos, por las afirmaciones de otros historiadores que, como Sänchez-Albornoz, sostienen que el aumento de la producción se obtuvo por la adición de tierras y no por el crecimiento de los rendimientos del suelo o de la mano de obra. Asimismo, en los estudios de Tortella sobre la agricultura española publicadas desde finales de los setenta late la idea del estancamiento tecnológico, según la cual la expansión del producto dependía más de la utilización de mayor cantidad de factores productivos (tierra y trabajo) que del aumento de la productividad de alguno de éstos 4 .

Durante la década de los ochenta se dejan oir las primeras dudas sobre el estancamiento secular de la productividad agraria en el siglo XIX 5. En 1988, Prados, al presentar los resultados de un método indirecto para estimar la demanda del producto agrario durante el siglo XIX, afirma que en el periodo 1800-1910 la productividad de la mano de obra creció a un ritmo anual del 0,4 por 100 y que la productividad de la tierra - superficie cultivada - lo hizo a una tasa anual acumulativa entre el 0,2 y un 0,6 debida en gran medida a la mejora de los rendimientos durante la segunda mitad del siglo XIX ${ }^{\circ}$. Una visión alternativa es la ofrecida por Simpson a través de la via del consumo, para quien los cambios significativos en la productividad de la tierra y de la mano de obra tendrían que esperar hasta el siglo $\mathrm{xx}{ }^{7}$.

Vicens Vives (1972), pp. 585.586

Sanchez-Albornoz (1977), p. 19, y Tortella (1981), p. 42.

'GEHR (1983), pp. 225-242, y también se pueden consultar las tesis doctorales de los miem. bros de este grupo. En la misma linca. Garrabou y Sanz Fernández (1985), pp. 96-108, aseguran que los rendimientos agricolas crecieron a finales del siglo XIX de una forma nada desdeñable.

- Prados de la Escosura (1988), pp. 114.115.

Simpson (1989), p. 381. 


\section{LAS FUENTES}

La principal dificultad a la hora de afrontar esta investigación consistia en la inexistencia de fuentes cuantitativas que nos informaran sobre las producciones y las productividades agrarias para todo el territorio nacional, hasta las primeras publicaciones de la Junta Consultiva Agronómica a finales del siglo $\mathrm{XIX}$. Este problema ha sido parcialmente resuelto con el empleo de los Cuadernos Generales de la Riqueza (1818-1820), fuente prácticamente desconocida en los estudios de historia agraria y que se utiliza por primera vez para conocer las productividades agricolas ${ }^{8}$, puesto que los ciento setenta y un pueblos para los que se ha localizado su Cuaderno - repartidos por trece provincias - constituyen un muestreo que ha permitido calcular los rendimientos medios de los principales cultivos para 1818 en España.

La caótica situación de la Hacienda durante el reinado de Fernando VII le condujo a intentar en diversas ocasiones reformar la fiscalidad real. El intento más importante fue el protagonizado por el ministro Martín de Garay, que con el Real decreto de 30 de mayo de 1817 establece la Contribución General del Reino, que consistía en la abolición de las rentas provinciales y su sustitución, en el medio rural, por un nuevo impuesto directo y proporcional al valor de la producción agraria - no de la propiedad - de cada contribuyente. Su puesta en marcha requería conocer el producto de las distintas ramas productivas del pais, y dado que el «Censo de Frutas y Manufacturas» de 1799 presentaba serias deficiencias, decide que se inicien los trabajos para la formación de una Estadistica del Reino que estaria compuesta por dos documentos: los Apeos y valuaciones generales del capital y productos especificos de todas las tierras, edificios y propiedades, y los Cuadernos Generales de la Riqueza de todos los pueblos de España, menos de las provincias vascas y Navarra, que gozaban de un régimen fiscal especifico.

El Cuaderno General de la Riqueza de cada población se divide en riqueza territorial, industrial y comercial, estando el primer apartado dedicado a todos los aspectos referentes a la agricultura, la ganadería y los edificios. Comienza haciendo una relación de la extensión y valor de todas las tierras que poseia en propiedad y en renta cada uno de los individuos del lugar, a continuación se anotaba la superficie dedicada a cada cultivo, el volumen de producción obtenida y su valor, del cual se descontaban los capitales anticipados a fin de conocer el montante sobre el que habria de imponerse la contribu.

\footnotetext{
* Una primera presentación de esta fuente está realizada en Santoveña Setien y Gutiérrez Bringas (1991).
} 
ción. De modo análogo se procedía con la ganaderia y los edificios, diferenciando entre los se destinaban a vivienda y a producción. Al final del Cuaderno se añadía un resumen donde se recogian los datos sobre la extensión de cada tipo de tierra, volumen y valor de sus productos, número de cabezas de ganado, valor de éstas y de su producción, así como, el número de edificios y su renta, al igual que se consignaban las rentas generadas por los oficios y otras actividades económicas desarrolladas en el pueblo.

Bien por medio del vaciado de la información vecino a vecino, o bien con el resumen del Cuaderno, cuando éste se ha conservado, es posible conocer la superficie sembrada que ocupaba cada cultivo, incluida la dejada en barbecho y en erial temporal, y la cantidad bruta cosechada, pudiendo de esta manera calcular la productividad media de los diferentes productos en cada lugar.

El desconocimiento sobre la localización de este fondo documental ha obligado a una lenta y laboriosa busqueda por más de una treintena de archivos municipales y provinciales de diversas regiones dando como resultado el hallazgo de los Cuadernos Generales de la Riqueza de 171 pueblos de trece provincias españolas (trece en Alicante, ocho en Barcelona, uno en Burgos, trece en Cádiz, cuarenta y cuatro en Cantabria, uno en Castellón, diecinueve en Córdoba, dos en Jaén, cuarenta y cinco en Madrid, diecisiete en Segovia, cinco en Sevilla, uno en Toledo y dos en Zamora), que forman posiblemente sólo una parte de los que en realidad se llegaron a elaborar, y de los cuales ciento cincuenta y cinco han podido ser utilizados en esta investigación.

Es pronto para emitir un juicio fundamentado sobre la fiabilidad de los datos extraidos de los Cuadernos Generales de la Riqueza, labor que constituirá el objeto de un próximo trabajo, pero de momento se puede afirmar que es similar a la de otras fuentes de caracter fiscal como el Catastro del Marqués de la Ensenada. Contrastados los datos de algunos pueblos con la información obtenida de las contabilidades privadas de fincas situadas en las mismas provincias $y$ en fechas cercanas, los resultados nos hacen ser optimistas en cuando al grado de fiabilidad de las cifras sobre la productividad de la tierra de los Cuadernos Generales de la Riqueza.

Además de la citada documentación se han utilizado otras fuentes que ofrecen una razonable seguridad en sus datos y que son sobramente conocidas por los historiadores. Por un lado, los Interrogatorios realizados en todos los pueblos de la Corona de Castilla entre 1750 y 1754 como parte esencial del Catastro del Marqués de la Ensenada, depositados en el Archivo General de Simancas, y por otro los datos publicados por la Junta Consultiva Agronómica en sus avances estadísticos de los años 1915 y 1923.

En las Respuestas Generales del Catastro, las preguntas doce y trece dan 
noticia sobre la producción por unidad de superficie sembrada, según los tipos de tierras y sus calidades, de los cereales, el viñedo, el olivar y con menos frecuencia de las leguminosas, to que ha permitido obtener una productividad media de cada una de las especies en los lugares para los cuales se habia localizado el correspondiente Cuaderno General de la Riqueza, con la única excepción de los situados en la Corona de Aragón, donde la Unica Contribución no tuvo vigencia.

A pesar de la búsqueda efectuada en el Archivo del Ministerio de Agricul. tura y en el Archivo General de la Administración, no ha sido posible localizar ni las memorias ni los informes que los ingenieros agrónomos de cada provin. cia remitieron a la Junta Consultiva Agronómica durante las primeras décadas del presente siglo y que sirvieron a este organismo de base para la elaboración de sus estadísticas. Estos informes debian proporcionar al menos la productividad de los distintos cultivos en cada una de las comarcas seleccionadas para realizar el muestreo con el cual estimar por el «procedimiento indirecto de los rendimientos», y debidamente ponderada la superficie sembrada, la produccion agraria provincial. Por desgracia estos informes han debido perderse durante la Guerra Civil, en la cual se destruyeron las bibliotecas del Consejo Agronómico y de la Escuela de Ingenieros Agrónomos, ignorándose el paradero del archivo de la Junta Consultiva Agronómica". Por lo tanto, nos hemos debido conformar con los datos provinciales sobre la productividad de la tierra publicados en los avances estadísticos para los años 1903/12 y 1922, y en los anuarios estadisticos de España de 1932 y 1933 para el año 1930.

La utilización de dos fuentes elaboradas con anterioridad al establecimiento definitivo del sistema métrico decimal en España - Catastro de Ensenada y Cuadernos Generales de la Riqueza - ha obligado a convertir las medidas tradicionales para hacer posible la comparación de resultados. Para solucionar este problema se ha recurrido al método que ofrece mayor seguridad, y que no es otro que el realizar la conversión de las medidas de superficie de cada uno de los pueblos con la ayuda de la información metrológica contenida en las Respuestas Generales del Catastro de Ensenada, concretamente su respuesta numero nueve, que permite conocer la medida o medidas de superficie usadas en cada uno de ellos (fanegas, obradas, aranzada, etc.) y su equivalencia a las unidades castellanas (pies, varas y estadales), y, por lo tanto, su traducción a hectareas ${ }^{10}$. La mayor homogeneidad de las antiguas medidas de capacidad en

"(1948) «Las publicaciones de la "Junta Consultiva Agronomica"», p. 227, y GEHR (1991), p. 29.

11) Para los pueblos de la Corona de Aragón se ha recurrido a la bibliografia, a la documentacion de archivo, $y$ en algunos casos, a las noticias que sobre medidas contienen los propios Cuadernos. 
las que se expresan las producciones agricolas de los pueblos analizados ha hecho posible tomar los valores de las medidas castellanas y convertirlas en hectólitros. Para transformar éstos en quintales métricos se han utilizado unos coeficientes, distintos según los productos, basándonos en la información proporcionada por una memoria de Eduardo Abela sobre la agricultura madrileña de mediados del siglo XIX ${ }^{11}$.

\section{LA TENDENCIA A LARGO PLAZO DE LA PRODUCTIVIDAD DE LA TIERRA EN ESPAÑA (1752.1930)}

Con las fuentes anteriormente descritas se ha pretendido reconstruir una tendencia a largo plazo de la productividad de la tierra, tanto de las superficies sembradas como de las cultivadas, entre los siglos xvill al $\mathrm{xx}$ tomando para ello los datos de los años 1752, 1818, 1903/12, 1922 y 1930.

\subsection{El estancamiento de la productividad de la tierra (1752-1818)}

A partir de las productividades medias de los cultivos de 145 pueblos en el año 1752 y 161 en 1818 (véanse los cuadros del apéndice estadistico), que representan una superficie agraria de mas de 350.000 hectáreas distribuidas en trece provincias de diferentes regiones, se ha calculado la producción por unidad de superficie para la agricultura española entre estas dos fechas de la manera siguiente.

Salvo casos excepcionales, las Respuestas Generales del Catastro de Ensenada no especifican las superficies sembradas de cada uno de los distintos cereales y leguminosas, por lo que se ha deducido la productividad media nacional de cada cultivo como una media simple de las productividades de todos los pueblos de la muestra. Si ofrece en cambio la extensión de tierras planta. das de viñedos y olivares - respuesta número diez- por lo que, conocido su

\footnotetext{
"Abela (1876), pp. 33 y 36 . En dicha memoria las producciones agricolas se manifiestan en
hectolitros y en kilogramos, lo que ha permitido obtener los siguientes coeficientes para cereales
y leguminosas $(100$ litions $y$ leguminosas (100 litros):
}

\begin{tabular}{|c|c|c|c|}
\hline Trigo & $78 \mathrm{Kgs}$. & Garbanzos & $78 \mathrm{Kgs}$ \\
\hline Cebada & $58 \mathrm{Kgs}$. & Habas & $78 \mathrm{Kgs}$ \\
\hline Centeno & $72 \mathrm{Kgs}$. & Judias & $77 \mathrm{Kgs}$ \\
\hline Avena & $46 \mathrm{Kgs}$. & Guisantes & $72 \mathrm{Kgs}$ \\
\hline Maiz . & $76 \mathrm{Kgs}$. & Lentejas & $.83 \mathrm{Kgs}$ \\
\hline
\end{tabular}


rendimiento segun las distintas calidades del terreno, se pueden averiguar las producciones medias de vino y de aceite, y por lo tanto calcular la productividad de estos dos cultivos como media ponderada del conjunto de los pueblos.

\section{CUADRO 1}

España 1752-1930. Productividad superficies sembradas ( $\mathrm{Qm} / \mathrm{ha}$ )

(números indices $1818=100$ )

\begin{tabular}{|c|c|c|c|c|c|}
\hline & 1752 & 1818 & $1903 / 12$ & 1922 & 1930 \\
\hline Trigo & $5,0(100)$ & $5,0(100)$ & $9,0(180)$ & $8.8(176)$ & $9,1(182)$ \\
\hline Cebada ..... & $7,1(109)$ & $6,5(100)$ & $11,8(181)$ & $11,7(180)$ & $12,8(197)$ \\
\hline Centeno ............... & $3,0(97)$ & $3,1(100)$ & $6.7(216)$ & $8,0(258)$ & $8,8(284)$ \\
\hline Avena & $4,8(171)$ & $2,8(100)$ & $9,2(328)$ & $8,4(300)$ & $10,4(371)$ \\
\hline Maiz & $12.3(189)$ & $6,5(100)$ & $14,6(224)$ & $15,2(234)$ & $17,6(270)$ \\
\hline Cereales ......... & $5,2(98)$ & $5,3(100)$ & $9,5(179)$ & $9.6(181)$ & $10,3(194)$ \\
\hline Garbanzos & $4,4(151)$ & $2,9(100)$ & $4,7(162)$ & $5,7(196)$ & $3,9(134)$ \\
\hline Habas ................. & $5,9(137)$ & $4,3(100)$ & $7,8(181)$ & $9,2(214)$ & $7,3(169)$ \\
\hline Guisantes ............. & - & $4,0(100)$ & $5,4(135)$ & $5,0(125)$ & $4.0(100)$ \\
\hline Judias ..... & $0.7(70)$ & $1,0(100)$ & 6,516501 & $8,2(820)$ & $8,6(860)$ \\
\hline Algarrobas & $2,6(173)$ & $1.5(100)$ & $6.3(420)$ & $5,8(386)$ & $5,0(333)$ \\
\hline Almortas .............. & $2,7(79)$ & $3,4(100)$ & $6,2(182)$ & $6,8(200)$ & - \\
\hline Alverjones .............. & $4,0(117)$ & $3.4(100)$ & $6.5(191)$ & $7,4(217)$ & - \\
\hline Yeros. & $3.5(73)$ & $4,8(100)$ & $6,3(131)$ & $8,5(177)$ & - \\
\hline Leguminosas & $3,5(145)$ & $2,5(100)$ & $6,3(252)$ & $7,1(286)$ & $5,8(232)$ \\
\hline Viñedo (a) ...... & $6,1(100)$ & $6,1(100)$ & $12,7(208)$ & $21,0(328)$ & $12,7(208)$ \\
\hline Olivar (a) ............... & $1.4(200)$ & $0,7(100)$ & $1,4(200)$ & $2,5(357)$ & $0,4(57)$ \\
\hline
\end{tabular}

Fufntes: Elaboracion a partir de los cuadros A.1 al A.9, los avances de 1915 y 1923 y los Anuarios de 1932 y 1933.

(a) $\mathrm{Hl} / \mathrm{ha}$

De igual manera se ha operado con la información de 1818 proveniente de los Cuadernos Generales de la Riqueza, con la única diferencia de que en este caso ha sido posible ponderar las productividades de todos los cultivos para obtener la media nacional, ya que esta fuente proporciona las superficies sembradas y las cantidades de cosechas de cereales y leguminosas en todos los pueblos ${ }^{12}$.

12 Por falta de espacio, en este trabajo se han tenido que omitir todos los datos de las productividades de las leguminosas y dejarlas reducidas a la media de todas ellas en cada lugar. 
En cuanto a la productividad del sistema cereal en las superficies sembradas en 1752, se ha hallado como la media aritmética de todos los casos en los que cuenta con los rendimientos de cereales y leguminosas, mientras que para ponderar la media nacional correspondiente a las superficies cultivadas, que nos aporta la fuente, ha sido necesario calcular previamente de la producción media anual de cada lugar utilizando los datos del propio Catastro de Ensenada ${ }^{13}$.

Restando de la superficie cultivada la extensión ocupada por el barbecho $y$ el erial temporal, que viene determinada por el sistema de cultivo de cada pueblo, se obtiene un aproximación a la superficie sembrada, y por lo tanto, es posible conseguir la media ponderada de la relación entre estas dos superficies ${ }^{14}$.

La labor se ve facilitada para 1818 por la propia fuente, que suministra directamente toda la información necesaria para el cálculo de las medias ponderadas a nivel nacional de las productividades del sistema cereal.

Todas estas operaciones han dado como resultado las cifras de los cuadros 1 y 2 , para los años 1752 y 1818 , cuyos resultados se resumen a continuación.

Entre estas dos fechas los rendimientos de los cereales en España, y en particular los del trigo, no experimentaron ningún cambio, al igual que debió suceder durante siglos hasta la segunda mitad del XIX. El trigo, con una productividad media de 5 quintales por hectárea, representaba en 1818 el 65 por 100 de la producción y el 70 por 100 de la superficie destinada a los cereales. Le seguian en importancia los cereales más rentables, la cebada y el maiz, que oscilaban entre 6 y $7 \mathrm{qm} / \mathrm{ha}$., seguidos por el centeno y la avena con unos ren. dimientos de 3 o $4 \mathrm{qm} / \mathrm{ha}$. Los $12,3 \mathrm{qm} / \mathrm{ha}$. del maiz en 1752 están sobrevalorados al incluir sólo la información de Cantabria, donde este cultivo presenta unos rendimientos muy superior a la media, frente a 6,5 de 1818 , donde se contabilizan las producciones de Barcelona, Castellón y Alicante.

Parece que estas productividades pueden ser generalizables a toda España, como lo constata el hecho de que estos valores sean muy semejantes a los obtenidos por Amalric a partir de los rendimientos de las tierras de segunda calidad de 540 pueblos de la comunidad de Castilla-León a mediados del siglo XVIII: trigo $5,8 \mathrm{qm} / \mathrm{ha}$., cebada $6,9 \mathrm{qm} / \mathrm{ha}$. y centeno $5,2 \mathrm{qm} / \mathrm{ha} .{ }^{15}$.

Por lo que respecta a la productividad de las leguminosas, su diferencia se debe más a una insuficiencia de las fuentes que a una desigualdad real, ya que

\footnotetext{
13 Para ello se ha seguido el método expuesto por Amalric y Brumont (1975), pp. 228-229, y

-amarero Bullón (1984)

it Otro método más rapido para obtener el mismo resultado consiste en dividir la producti-

vidad de la superficie sembrada entre la productividad de la superficie cultivada.

Amalric (1990), pp. 311-316.
} 


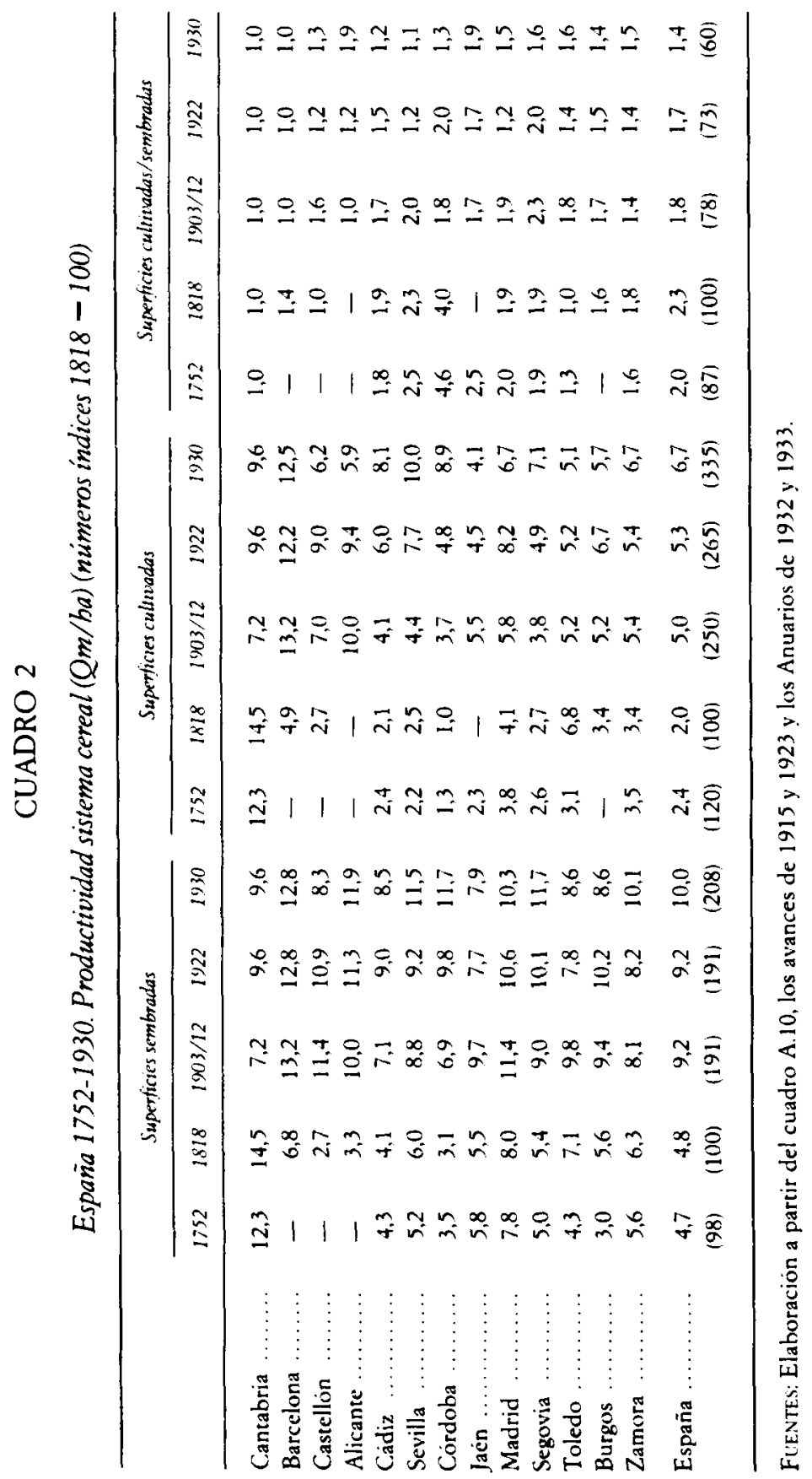


los rendimientos obtenidos de las Respuestas Generales están estimados al alza puesto que sólo se facilitan las productividades de las leguminosas en aquellos pueblos donde estos cultivos tenian unas producciones significativas, y por tanto también sus rendimientos. De aqui, el que la productividad media haya sido calculada con un menor número de casos que en 1818 .

La evolución del sistema cereal permite comprobar también cómo la productividad de la tierra se mantuvo estancada durante la segunda mitad del siglo XvIII en unos niveles que no sobrepasaron los $5 \mathrm{qm} / \mathrm{ha}$. en las superficies sembradas y de la mitad en las cultivadas, debido al sistema de cultivo dominante en España, que no era otro que el de «año y vez», que suponia dejar en descanso aproximadamente el 55 por 100 de las tierras dedicadas a los cereales y las leguminosas ${ }^{16}$. El aumento del barbecho entre un 10 ó un 15 por 100 explicaria la disminución de los rendimientos en las tierras cultivadas en un 20 por 100 y el descenso de la producción agricola en las dos primeras décadas del siglo XIX.

Por su parte, el viñedo manifiesta la misma tendencia al coincidir los resultados de ambas fuentes. Los $6,1 \mathrm{Hl} /$ ha. se ven ratificados por el trabajo de Huetz de Lemps sobre las superficies y las producciones viticolas en el noroeste de España durante el siglo XVIII, gracias al cual se ha podido calcular la productividad media de 177.093 hectáreas de viñedo en 1306 pueblos de catorce provincias - con una producción de $1.193 .590 \mathrm{hls}$. de vino- con el resultado de $6,7 \mathrm{hl} / \mathrm{ha}$. en $1752^{17}$.

Los bajos rendimientos del olivar en 1818 se deben explicar por las malas cosechas habidas durante la segunda década del siglo XIX, con el consiguiente descenso de la cantidad de aceite elaborado en las villas productoras de las provincias de Cádiz, Córdoba, Jaén y Sevilla. Así parece demostrarlo el ejemplo de Sanlúcar de Barrameda (Cádiz), donde las 363 hectáreas plantadas con 12.000 olivos nada produjeron por el mal estado en que se hallaban todos los olivos de este término desde hacia siete años, frente a los 663 hectólitros de aceite extraídos de las 313 hectáreas en esta misma localidad en 1752. Son, por tanto, los datos del Catastro los más representivos, con una productividad media de $1,4 \mathrm{HI} / \mathrm{ha}$. que se mantuvo a lo largo del xvill, como lo confirma la unica información disponible sobre las cuentas de una hacienda olivarera, la ción media por hectárea entre 1739 y 1774 fue de 2 hectólitros:

\footnotetext{
de las En 1818 la superficie ocupada por los cereales y las leguminosas suponian el 75 por 100

17 sembradas y el 38 por 100 de las tierras cultivadas.

17 Huetz de Lemps (1967), tomo II, pp. 869.917.
} 


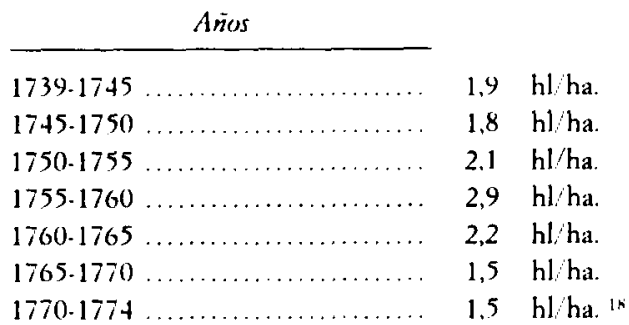

En definitiva, todo lo dicho hasta el momento confirma la idea dominante en la literatura del estancamiento de la productividad de la tierra en los cultivos más importantes de la agricultura española durante entre 1752 y 1818 con indiferencia de la provincia analizada, asi como su bajo nivel con respecto a las conseguidas en otros paises europeos. Comparados los rendimientos del trigo en España con las estimaciones de Bairoch para 1800 resulta que, menos Rusia, con 5,4 qm/ha., el resto de la Europa occidental presenta una productividad superior en un 40 por 100 a la española (Alemania, $10 \mathrm{gm} / \mathrm{ha}$.; Bélgica, 13,5 qm/ha.; Holanda, $14 \mathrm{qm} / \mathrm{ha}$.; Francia, $8,5 \mathrm{qm} / \mathrm{ha}$.; Italia, 7,3 qm/ha., o el Reino Unido con $13,6 \mathrm{qm} / \mathrm{ha}$.). Para el resto de los cereales la diferencia oscila entre el 30 por 100 del maiz o el 55 por 100 de la avena y el centeno, mientras que únicamente la cebada muestra unos rendimientos muy semejantes a la media europea ${ }^{19}$.

Con excepción de Pérez Garcia, que defiende para Galicia un «progreso de los rendimientos en la segunda mitad del siglo Xvill» ${ }^{20}$, el resto de los historiadores que han abordado este tema utilizando las contabilidades de explotaciones agrícolas privadas son unánimes en su conclusión de que la productividad de la tierra permaneció estancada hasta entrado el siglo xIX, confirmando no sólo la tendencia, sino también los valores de los rendimientos obtenidos, y por tanto, la fiabilidad de las fuentes fiscales empleadas.

Para Garcia Sanz, en Castilla uno se dio ningún cambio significativo en el nivel de los rendimientos» en el siglo Xvil ${ }^{21}$. Sirviéndose de los libros de cuentas de una granja de la provincia de Segovia, precisa sus productividades para el periodo $1739-1767$ en $5,3 \mathrm{qm} / \mathrm{ha}$. para el trigo, $13,7 \mathrm{qm} / \mathrm{ha}$. para la cebada, $2,0 \mathrm{qm} /$ ha. para el centeno y $6,4 \mathrm{qm} / \mathrm{ha}$. para la media de los cereales 22 .

\footnotetext{
is Ponsot (1985), pp. $180 \times 190$.

14 Bairoch (1989), pp. 320 y 322.

21 Pérez Garcia (1979), p. 197

2 Garcia Sanz (1986), p. 157.

22 Garcia Sauz (1985), p. 211.
} 
Al mismo resultado llegan Brumont, a propósito de una finca burgalesa donde los rendimientos no evolucionaron entre 1630 y $1835^{23}$, y Gonzalo Anes, después de analizar las explotaciones agrícolas de Aranjuez (Madrid), donde se produce «un estancamiento de los rendimientos por unidad de superficie y por fanega sembrada» como lo demuestran los datos siguientes ${ }^{24}$ :

\begin{tabular}{|c|c|c|c|}
\hline & Trigo & Cebada & Cereales \\
\hline 1768.77 & 8,1 & 14,6 & 11,0 \\
\hline $1778.87 \ldots \ldots \ldots \ldots \ldots$ & 6,9 & 14,0 & 9,1 \\
\hline $1788.95 \ldots \ldots \ldots \ldots \ldots$ & 8.9 & 10,1 & 9,4 \\
\hline 1768.95 & 7,6 & 13,9 & 9,4 \\
\hline
\end{tabular}

A Llopis Agelán, que estudia la economía monástica de finales del Antiguo Régimen en Extremadura, no le cabe ninguna duda de que, aparte de ser baja, «a productividad agricola de las granjas del monasterio (de Guadalupe) no experimentó el más minimo avance a lo largo de la segunda mitad del siglo XVIII, 25. Y los cinco cortijos de la campiña cordobesa estudiados por Ponsot en los siglos XVII al XIX lo ratifican

\begin{tabular}{|c|c|c|c|}
\hline & Trigo & Cebada & \\
\hline $1611-1650$ & 3,0 & 2,5 & $\mathrm{qm} / \mathrm{ha}$. \\
\hline 1651.1700 & 3,6 & 4,1 & qm/ha. \\
\hline 1701.1750 & 4,5 & 6,6 & $\mathrm{qm} / \mathrm{ha}$. \\
\hline 1751.1800 & 4,1 & 5,8 & $\mathrm{qm} / \mathrm{ha}$. \\
\hline $1801 \cdot 1841$ & 4,0 & 6,4 & $\mathrm{qm} / \mathrm{ha} \cdot{ }^{2 \mathrm{t}}$ \\
\hline
\end{tabular}

Fuera de la península el fenómeno se repite de la misma manera como sucede en Tenerife y en Mallorca, donde "podemos comprobar perfectamente cómo los rendimientos reales no aumentaron en el transcurso del siglo XVIIIs 27. Y por último, una recopilación de la información aportada por la bibliografía sobre los rendimientos medios por semilla — producción entre cantidad de simiente - en los siglos XVI al XIX ha permitido constatar de nuevo la tendencia al estancamiento de la producción por hectárea.

\footnotetext{
23 Brumont (1979), pp.396-399.

24 Anes (1974), pp. 193 y 194.195.

25 Llopis Agelán (1980), p. 351.

27 Ponsot (1982), pp. 439-443.

Núñez Pestano (1984) y Juan Vidal (1978), p. 52.
} 


\begin{tabular}{|c|c|c|c|c|}
\hline & Trige & Cebada & Centenos & Avena \\
\hline Siglo xv' & 5,4 & 6,1 & 5.4 & 4,3 \\
\hline Siglo xirt ......... & 4,0 & 5,5 & 3,3 & 2.6 \\
\hline Siglo xvitt ........ & 5,1 & 6.3 & 4,0 & 3,4 \\
\hline 1." mirad & 5,2 & 5,7 & 4,3 & - \\
\hline 2.4 mitad ..... & 5,0 & 6.7 & 3,8 & 3,4 \\
\hline Siglo $x i x\left(1 .{ }^{4}\right.$ mitad $), \ldots \ldots \ldots$ & 5,4 & 5,9 & 4,4 & -28 \\
\hline
\end{tabular}

\subsection{El crecimiento de la productividad de la tierra (1818-1930)}

Las superficies y las producciones agrarias a nivel provincial de los avances estadisticos de 1915 y 1922, además de los anuarios estadísticos de 1932 y 1933, han proporcionado el resto de la información precisa para estudiar la evolución a largo plazo de los rendimientos agrícolas en ausencia de otra documentación con estos datos desagregados por pueblos o por comarcas. Por ello, a la hora de proponer una productividad media para todo el pais, ésta se ha calculado como media ponderada solamente de las trece provincias a las que corresponden los pueblos del muestreo efectuado para 1752 y 1818 (veánse cuadros 1 y 2 ), siendo el resultado unos rendimientos muy próximos a la media nacional - para las cuarenta y nueve provincias - de cada cultivo, lo que garantiza su representatividad, como se puede observar en las siguientes cifras:

\begin{tabular}{|c|c|c|c|}
\hline Años & $1903 / 12$ & 1922 & 1930 \\
\hline Trigo . & 8,5 & 8,9 & 9,2 \\
\hline Cebada & 9,7 & 10,7 & 12,6 \\
\hline Centeno $\ldots \ldots \ldots \ldots \ldots \ldots \ldots \ldots$ & 6,7 & 9,3 & 9,8 \\
\hline Avena $\ldots \ldots \ldots \ldots \ldots \ldots \ldots$ & 6,9 & 7,5 & 9,3 \\
\hline Maiz & 13,3 & 15,9 & 15,3 \\
\hline Cereales $\ldots \ldots \ldots \ldots \ldots \ldots \ldots \ldots$ & 8,9 & 9,5 & 10.4 \\
\hline Leguminosas & 5,9 & 6,4 & 7,0 \\
\hline Sistema cereal ss ..... & 8,6 & 10,0 & 10,9 \\
\hline Sistema cereal sc $\ldots \ldots \ldots \ldots \ldots$ & 4,8 & 5,7 & 6,4 \\
\hline Sup culti./sembr. ................ & 1,7 & 1,7 & 1,6 \\
\hline Vinedo $(\mathrm{hl} / \mathrm{ha}) \ldots \ldots \ldots \ldots \ldots \ldots$ & 12,0 & 17.6 & 12,8 \\
\hline Olivar $(h \mathrm{~h} / \mathrm{ha}$ a) $\ldots \ldots \ldots \ldots \ldots \ldots$ & 1,5 & 2,2 & 0,6 \\
\hline
\end{tabular}

28 Elaboracion a partir de Brumont (1979) y (1985), Floristan Imizcoz (1982), Garcia Sanz (1985) y (1986), Garrabou (1978), Juan Vidal (1978), Llopis Agelan (1980) y Vilar (1967). 
A la vista de los datos aportados por las fuentes, se obtiene la impresión de que la productividad de la tierra en España creció a lo largo del siglo XIX. Más concretamente, este crecimiento habría que situarlo en el último tercio del siglo, para mantener su nivel durante las primeras décadas del $\mathrm{xx}$ en las superficies sembradas, en tanto que las cultivadas de cereales $y$ leguminosas incrementan sus rendimientos por una progresiva reducción del barbecho y los eriales hasta la llegada de la II República.

\section{CUADRO 3}

España 1752-1930. Productividad superficies sembradas y cultivadas medida agregada (calorias/ha) (nümeros indices 1818-100)

\begin{tabular}{|c|c|c|c|c|c|c|c|c|c|c|}
\hline \multirow{2}{*}{7} & \multicolumn{5}{|c|}{ Superfictes sembradas } & \multicolumn{5}{|c|}{ Superficies cultiviadas } \\
\hline & 1752 & 1818 & $1903 / 12$ & 1922 & 1930 & 1752 & 1818 & $1903 / 12$ & 1922 & 1930 \\
\hline Cantabria & 87 & 100 & 85 & 113 & 105 & 87 & 100 & 85 & 113 & 105 \\
\hline Barcelona & - & 100 & 153 & 169 & 148 & - & 100 & 186 & 203 & 179 \\
\hline Castellón ......... & - & 100 & 291 & 271 & 175 & - & 100 & 222 & 241 & 153 \\
\hline Alicante .......... & - & 100 & 174 & 259 & 260 & - & 100 & - & - & - \\
\hline Càdiz ......... & 97 & 100 & 172 & 219 & 196 & 111 & 100 & 190 & 275 & 339 \\
\hline Sevilla .......... & 69 & 100 & 122 & 142 & 141 & 28 & 100 & 157 & 258 & 261 \\
\hline Córdoba $\ldots \ldots$ & 120 & 100 & 124 & 347 & 269 & 197 & 100 & 434 & 640 & 639 \\
\hline Jaén ..... & 120 & 100 & 196 & 233 & 118 & - & 100 & - & - & - \\
\hline Madrid ......... & 62 & 100 & 139 & 140 & 126 & 89 & 100 & 137 & 192 & 154 \\
\hline Segovia ........... & 62 & 100 & 158 & 180 & 215 & 98 & 100 & 139 & 177 & 262 \\
\hline Toledo ....... & 95 & 100 & 340 & 275 & 282 & 61 & 100 & 200 & 206 & 192 \\
\hline Burgos ......... & 93 & 100 & 360 & 407 & 336 & - & 100 & 245 & 322 & 274 \\
\hline Zamora ........ & 84 & 100 & 126 & 128 & 165 & 100 & 100 & 156 & 158 & 202 \\
\hline España & 101 & 100 & 218 & 246 & 218 & 104 & 100 & 270 & 347 & $316^{24}$ \\
\hline
\end{tabular}

FutNTES: Elaboración a partir del Catastro del Marqués de la Ensenada, los Cuadernos Generales de la Riqueza, los Avances de 1915 y 1923, los Anuarios de 1932 y 1933 y las Tablas de composición de alimentos y Tablas cienificicas.

Mientras que el trigo y la cebada aumentan su productividad entre 1818 y $1903 / 12$ en un 70 por 100 , el resto de los cereales lo hacian en mayor propor. ción al duplicarse la del centeno y el maiz, y la avena hacerlo en una cuantía

\footnotetext{
${ }^{24}$ Flores de Lemus (1976) p. 427.
} 
algo superior. Al lado de este crecimiento del 70 por 100 de los cereales, las leguminosas multiplican por dos sus rendimientos; unos y otras conjuntamente lo hacen en las superficies sembradas entre un 80 y un 90 por 100. Sin embargo, tal vez donde mejor se comprueba la verdadera intensificación y progreso de la agricultura española durante el siglo XIX sea en el incremento de la productividad del sistema cereal en las tierras cultivadas entre un 135 a un 145 por 100 por la disminución del 10 al 20 por 100 de la superficie en barbecho. Esta reducción resulta semejante a la estimada por Flores de Lemus, que la sitúa en un 10 por 100 , y progresivamente irá descendiendo durante el presente siglo ${ }^{30}$.

Algo parecido ocurre con el viñedo, que duplica su productividad; en cambio, en el olivar sorprende que no halla experimentado cambios significativos en la producción por hectárea entre 1818 y la primera década del siglo XX.

El cuadro 3 se ha confeccionado siguiendo el mismo método empleado en los dos cuadros anteriores. Su objetivo es el de ayudar a conocer el crecimiento de la productividad de la tierra para el conjunto de la agricultura, para lo cual se han convertido las producciones a una unidad común - las calorías-, a fin de evitar en lo posible las desviaciones motivadas por los distintos rendimientos de cada cultivo.

Las cifras del cuadro muestran un progreso de la productividad de las tierras sembradas del 115 y al 120 por 100 entre 1818 y $1903 / 12$, y de las tierras cultivadas que lo hacen entre un 165 y un 170 por 100 , superando las estimaciones de Prados para el periodo 1800-1910, que los cifraba entre 20 a 60 por 100 y que llega al 78 por 100 cuando la obtiene por una estimación directa ${ }^{31}$. En ambos casos, y obtenida por métodos diferentes, se dibuja una tendencia de la evolución de la productividad de la tierra en la agricultura española durante el siglo xIX coincidente, aunque no en cuanto al grado de crecimiento ${ }^{32}$.

A pesar de este crecimiento de la productividad de la tierra, no disminuyó la distancia que separaba a los rendimientos medios de nuestra agricultura con los obtenidos por los países europeos más desarrollados, manteniéndose de esta manera el atraso en los campos españoles frente a sus competidores europeos. En 1910, la productividad del trigo español era de $8,5 \mathrm{qm} / \mathrm{ha}$., sólo infe-

30 Este brusco descenso en 1930 de la productividad de las superficies sembradas y cultivadas, medida en calorias, se debe a la importante caida de los rendimientos por hectárea del olivar.

31 Prados de la Escosura (1988), p. 129.

32 Realizada la conversión de la producción a precios constantes de 1818 y de 1922, los números indices resultantes concuerdan con estos porcentajes, oscilando para estos años entre 160 y 170 por 100 . Desgraciadamente, por falta de espacio no se pueden incluir estos cuadros. 
rior al de Rusia - 6,6 qm/ha. - y equivalente al italiano con 9,6 qm/ha.; el resto de paises obtenia unos rendimientos muy superiores: Bélgica, Holanda y Reino Unido, entre 21 y $25 \mathrm{qm} /$ ha.; Alemania, 18,5 qm/ha., y Francia, con 13,2 $\mathrm{qm} / \mathrm{ha}$., de tal manera que el trigo y la cebada presentaban una productividad media un 40 por 100 inferior a la media europea, el centeno y la avena el 50 por 100 y sólo el maiz alcanzaba unos niveles semejantes ${ }^{33}$. En definitiva, la productividad de la agricultura española quedaba por debajo de la de los paí. ses europeos avanzados en un 40 por 100 para los cereales, similar a la calculada por Bernal, para quien el diferencial no era inferior al 30 ó 40 por $100^{34}$.

\section{ARCHIVOS Y BIBLIOTECAS CONSULTADOS (abreviaturas)}
AHN
Archivo Histórico Nacional (Madrid).
AGS
Archivo General de Simancas (Valladolid).
AGDB Archivo General de la Diputación de Barcelona.
ARM Archivo Regional de Madrid.
AHPCa- Archivo Histórico Provincial de Cádiz.
AHPC Archivo Histórico Provincial de Cantabria
AHPCó Archivo Histórico Provincial de Córdoba.
AHPJ Archivo Histórico Provincial de Jaén.
AHPS Archivo Histórico Provincial de Segovia.
ADS Archivo Diocesano de Santander.
AHMC Archivo Histórico Municipal de Castellón.
AMAH Archivo Municipal de Alcala de Henares (Madrid).
AMCH Archivo Municipal de Chinchón (Madrid).
AMD Archivo Municipal de Denia (Alicante).
AMDH Archivo Municipal de Dos Hermanas (Sevilla).
AME Archivo Municipal de Ecija (Sevilla).
AMG Archivo Municipal de Getafe (Madrid).
AMM Archivo Municipal de Marchena (Sevilla).
AMPS Archivo Municipal de Poza de la Sal (Burgos)
AMS Archivo Municipal de Santander.
AMSe Archivo Municipal de Segovia.
AMSSR Archivo Municipal de San Sebastián de los Reyes (Madrid)
AMTT Archivo Municipal de Talavera de la Reina (Toledo).
BMS
Archivo Municipal de Toro y Tagarabuena (Zamora).
Biblioteca Municipal de Santander.

\footnotetext{
is Barbiero (1988), p. 118, y Bairorh (1989), pp. 320-322.

it Bernal (1991), p. 142.
} 
APÉNDICE ESTADÍSTICO

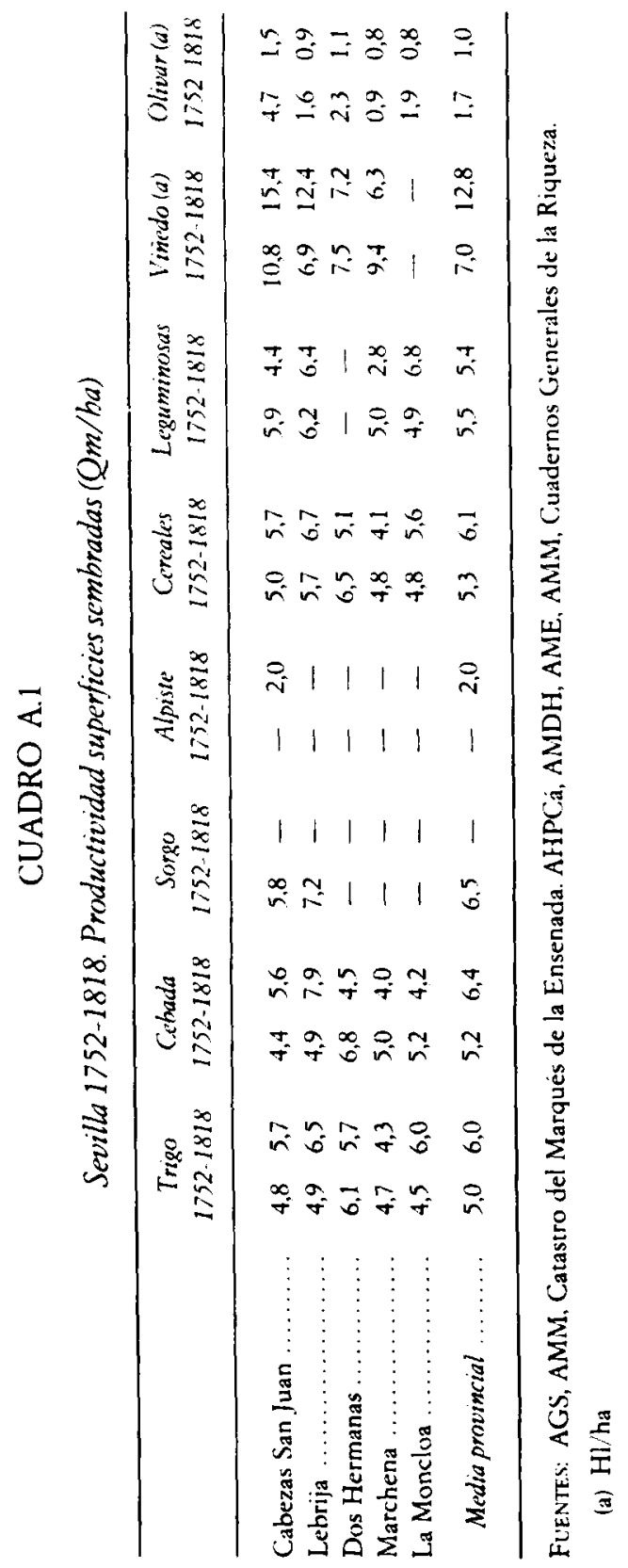




\section{CUADRO A.2}

Cantabria 1752-1818. Productividad superficies sembradas (Qm/ ha)

\begin{tabular}{|c|c|c|c|c|c|c|c|c|}
\hline \multirow[b]{2}{*}{ Santander $\ldots \ldots \ldots \ldots \ldots \ldots \ldots$} & \multicolumn{2}{|c|}{$\begin{array}{c}\text { Trigo } \\
1752-1818\end{array}$} & \multicolumn{2}{|c|}{$\underset{1752-1818}{\text { Maiz }}$} & \multicolumn{2}{|c|}{$\begin{array}{l}\text { lecgummosas } \\
17521818\end{array}$} & \multicolumn{2}{|c|}{$\begin{array}{l}\text { Vinedo }(a) \\
1752-1818\end{array}$} \\
\hline & 15,0 & $\cdots$ & 14.6 & 17.5 & 0.5 & 1,0 & 11,2 & 18,1 \\
\hline 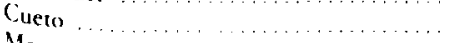 & 13.4 & - & 15.5 & 16,8 & 0.7 & 1,0 & 16,3 & 19,4 \\
\hline 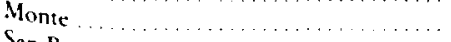 & - & & 11.8 & 17,5 & 0,4 & 1,1 & 11,5 & 23,3 \\
\hline 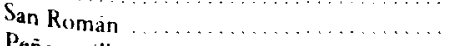 & - & & 13,3 & 18,0 & 0,4 & 0.9 & 13,4 & 10,2 \\
\hline$\ldots \ldots \ldots \ldots \ldots \ldots$ & 9,0 & - & 9,7 & 16,3 & 0,1 & 0,9 & 8.2 & 9,4 \\
\hline Liencres... & 7.0 & 9,0 & 7.8 & 11,6 & 1,0 & 1,0 & 4.5 & 10,7 \\
\hline Is $]_{\mathbf{a}} \ldots$ & 9.0 & - & 9.1 & 9,4 & 0,9 & 0.5 & 16,6 & 7.5 \\
\hline$\ldots \ldots \ldots \ldots \ldots \ldots \ldots \ldots$ &.- & - & 11,2 & 7.9 & 2,1 & 0.1 & 33,0 & 15.0 \\
\hline Villapresente $\ldots \ldots \ldots \ldots \ldots \ldots \ldots$ & - & - & 38.0 & 29.3 & 1.5 & 4,8 & - & $\cdots$ \\
\hline Villaverde $\ldots \ldots, \ldots \ldots \ldots \ldots \ldots \ldots$ & 4.7 & 8.7 & 7,5 & 11,4 & - & 0.9 & - & $\cdots$ \\
\hline Puente Viesgo Acs $\ldots \ldots \ldots \ldots \ldots \ldots \ldots \ldots$ & 14,3 & & 13,9 & 10,2 & - & 0.4 & 21,5 & - \\
\hline Presillas ........... & 5,0 & $-\cdot$ & 6,5 & 13,7 & 0,4 & 0,9 & & - \\
\hline ( & - & - & 8,4 & 11,6 & 0.4 & 0.4 & 6,0 & \\
\hline$\ldots \ldots \ldots \ldots \ldots \ldots$ & 14,3 & - & 15.5 & 12,0 & 1,1 & 1,1 & $\ldots$ & - \\
\hline$\ldots . .$. & 10,0 & -. & 9.7 & 9,4 & 0,3 & 0.4 & - & $\cdots$ \\
\hline$\ldots \ldots \ldots$ & 16,7 & - & 13.9 & 6,2 & 0.4 & 0,6 & - & \\
\hline Santiurde. & 23.8 & - & 18.6 & 21,0 & 1.1 & 3.5 & - & - \\
\hline San Marrin $\ldots$ & 16.7 & - & 21.7 & 6,1 & 0.5 & 0.4 & $\ldots$ & - \\
\hline 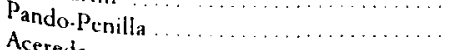 & - & - & - & 7.2 & - & 0.8 & $\cdots$ & - \\
\hline $\begin{array}{l}\text { Acereda } \ldots \ldots \ldots \ldots \ldots \ldots \ldots \ldots \ldots \ldots \ldots \ldots \ldots \ldots \ldots \ldots \ldots \\
\text { lruz }\end{array}$ & $\cdots$ & $\ldots$. & - & 7,1 & $\ldots$ & 0,6 & - & - \\
\hline 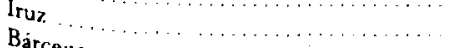 & 14,3 & 4.9 & 13.9 & 0,6 & 0.2 & 0,4 & - & - \\
\hline $\begin{array}{l}\text { Barcena } \\
\text { Vejoris }\end{array}$ & - & - & 8,5 & 7,0 & - & 0,4 & - & - \\
\hline$\cdots \cdots \cdots$ & - & $\ldots$ & 9,5 & - & 0,1 & $\ldots$ & - & .. \\
\hline Prases & 16.7 & - & 15,5 & 6,2 & 0.5 & 0,6 & 32,0 & - \\
\hline $\begin{array}{l}\text { Quintana } \\
\text { Espon }\end{array}$ & 11,9 & - & 15,5 & 5.1 & - & 0,2 & - & - \\
\hline Esponzues & 9,5 & - & 13,9 & 15,4 & - & 0,9 & -- & - \\
\hline$\ldots \ldots \ldots \ldots \ldots \ldots \ldots \ldots \ldots \ldots$ & 14,3 & 14,3 & 15.5 & 6,6 & 2,3 & 1.0 & 32,0 & - \\
\hline $\begin{array}{l}\text { San Vicente } \\
\text { Ontaned }\end{array}$ & 16.7 & - & 18.6 & 25,4 & 0,5 & 1,8 & - & - \\
\hline $\begin{array}{l}\text { Untaneda } \\
\text { Alceda }\end{array}$ & 13.5 & - & 13.2 & 13,6 & - & 1,6 & -.- & - \\
\hline $\begin{array}{l}\text { Alceda } \ldots \ldots \ldots \ldots \ldots \ldots \ldots \ldots \\
\text { Castillo }\end{array}$ & 9.5 & 一 & 13.9 & 12,6 & 一 & 1,2 & $\cdots$ & - \\
\hline $\begin{array}{l}\text { Castillo Pedroso } \ldots \ldots \ldots \\
\text { Borlena. S }\end{array}$ & 11.9 & - & 15,5 & 8,3 & - & 0,3 & - & - \\
\hline $\begin{array}{l}\text { Borlena-Salcedillo } \ldots \ldots \ldots \ldots \ldots \ldots \ldots \ldots \\
\text { Villegar }\end{array}$ & 14,3 & - & 18,6 & 6,0 & ..- & 0,6 & $\cdots$ & $\cdots$ \\
\hline $\begin{array}{l}\text { Illegar } \ldots \ldots \ldots \ldots \ldots \ldots \ldots \ldots \ldots \ldots \ldots \ldots \ldots \\
\text { Luena }\end{array}$ & 14,3 & - & 17,8 & 25,0 & 0,5 & 1,2 & - & - \\
\hline 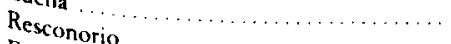 & - & - & 14.5 & 9,3 & - & 0,3 & - & $\ldots$ \\
\hline Entrambasmorio $\ldots \ldots \ldots \ldots \ldots \ldots \ldots \ldots \ldots \ldots$ & - & - & 11,8 & 7.3 & .. & -- & - & - \\
\hline Cañedo & - & - & 13,9 & $+2,6$ & $\cdots$ & 1,7 & - & - \\
\hline Santavan $_{\text {ando }} \ldots \ldots \ldots \ldots \ldots \ldots \ldots \ldots \ldots \ldots$ & 4,3 & 13.5 & 4.7 & 16,0 & - & 1,1 & - & - \\
\hline Valcaba & 4,6 & 8.0 & 8.8 & 8,4 & - & -- & - & - \\
\hline$S_{a n} M_{\text {artin }} S_{\text {ah }} \ldots \ldots \ldots \ldots \ldots \ldots \ldots$ & 4,2 & 8.7 & 6,2 & 11,2 & - & 0.7 & - & \\
\hline Valdicio-Calseca & 4,6 & 8.3 & 6,5 & 7,8 & - & - & - & - \\
\hline Medio-Calseca $\ldots \ldots \ldots \ldots \ldots \ldots \ldots$ & - & - & 8,4 & 3,8 & $\ldots$ & - & - & - \\
\hline Media provincial ..................... & 11.1 & 8.9 & 12.4 & 13,9 & 0.7 & 0,8 & 10.9 & 11.2 \\
\hline
\end{tabular}

FUENTR: AGS, AHPC, Catastro del Marqués de la Ensenada. AHPC, ADS, AMS, BMS, Cuadernos Generales de la Riqueza.

(a) $\mathrm{Hl} / \mathrm{ha}$ 


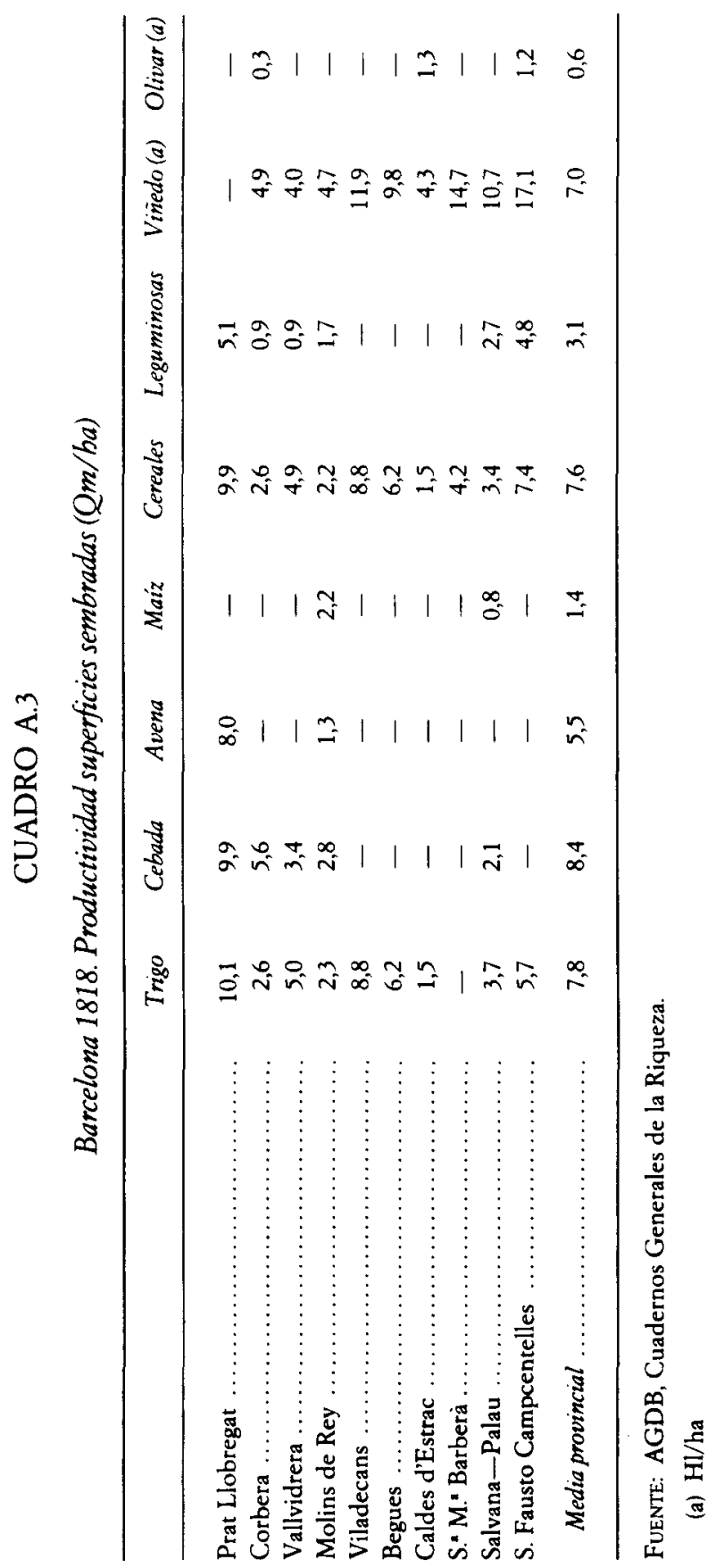




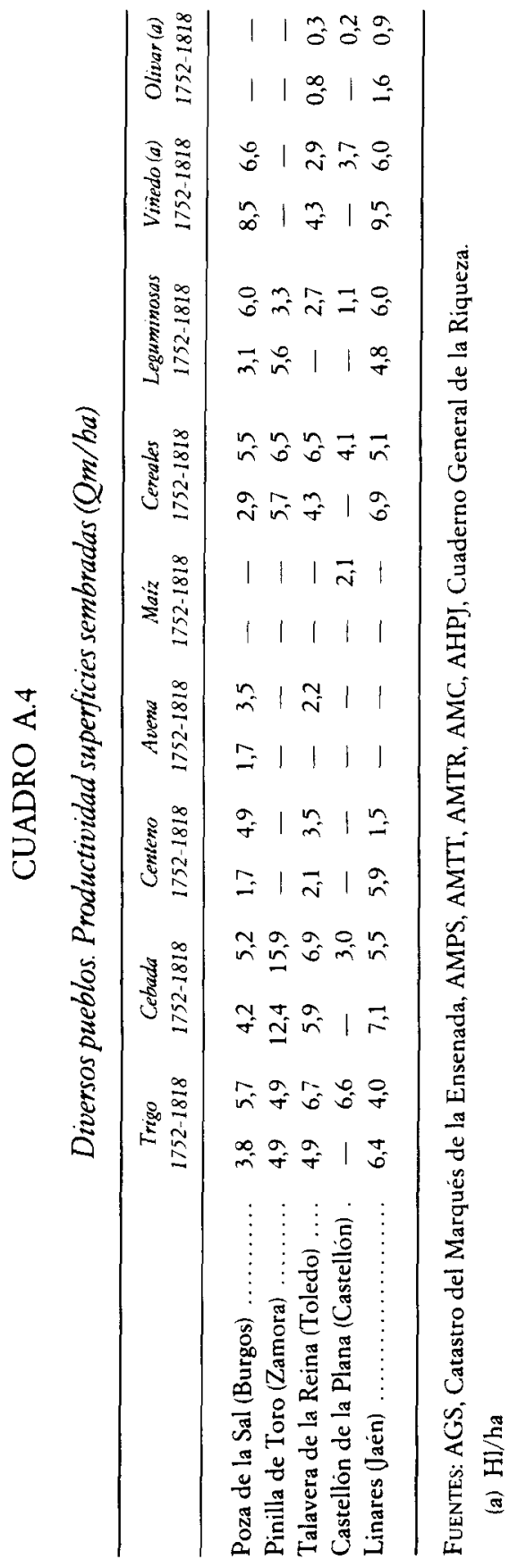




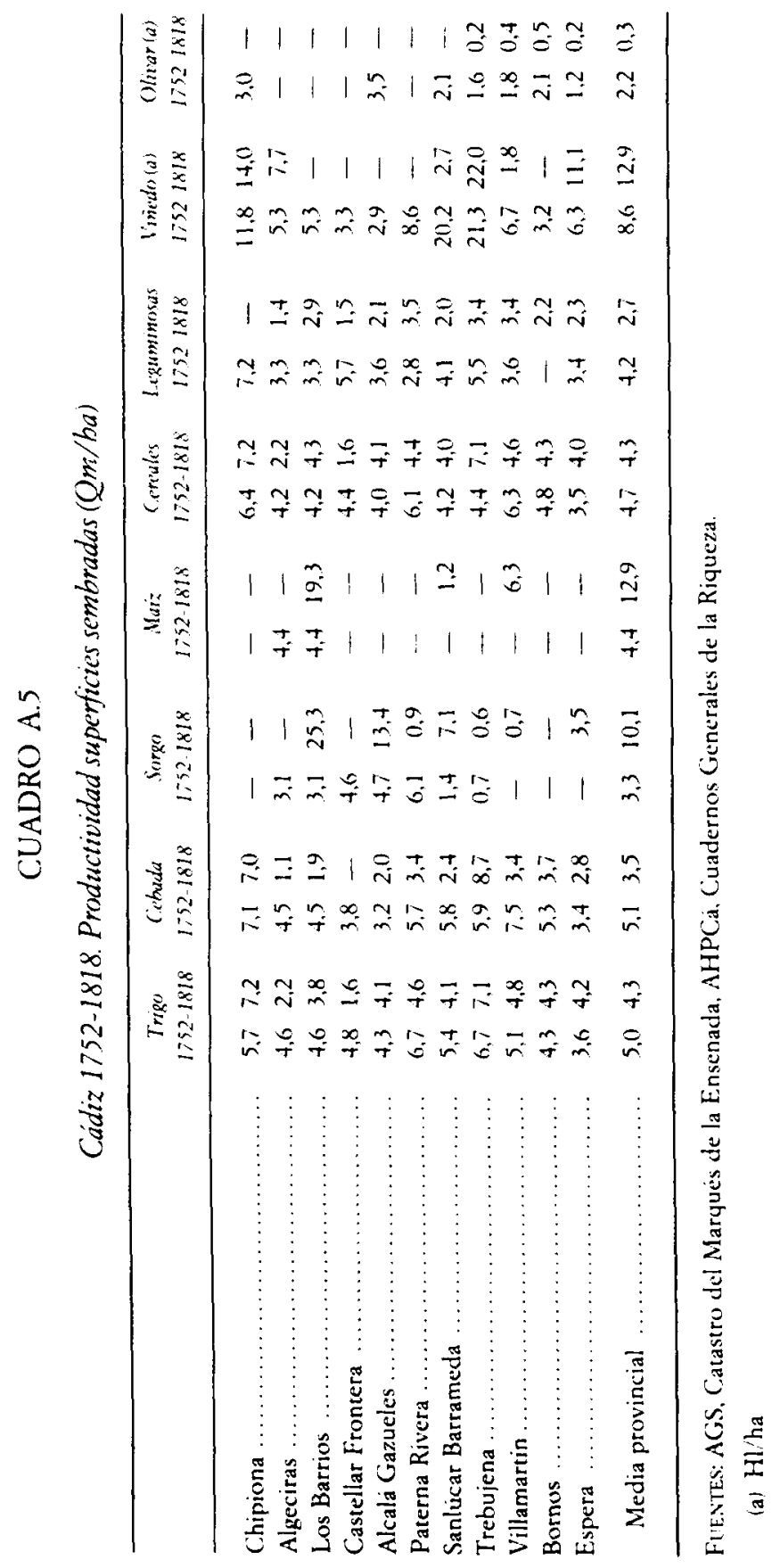




\section{CUADRO A.6}

Madrid 1752-1818. Productividad superficies sembradas ( $Q m / h a)$

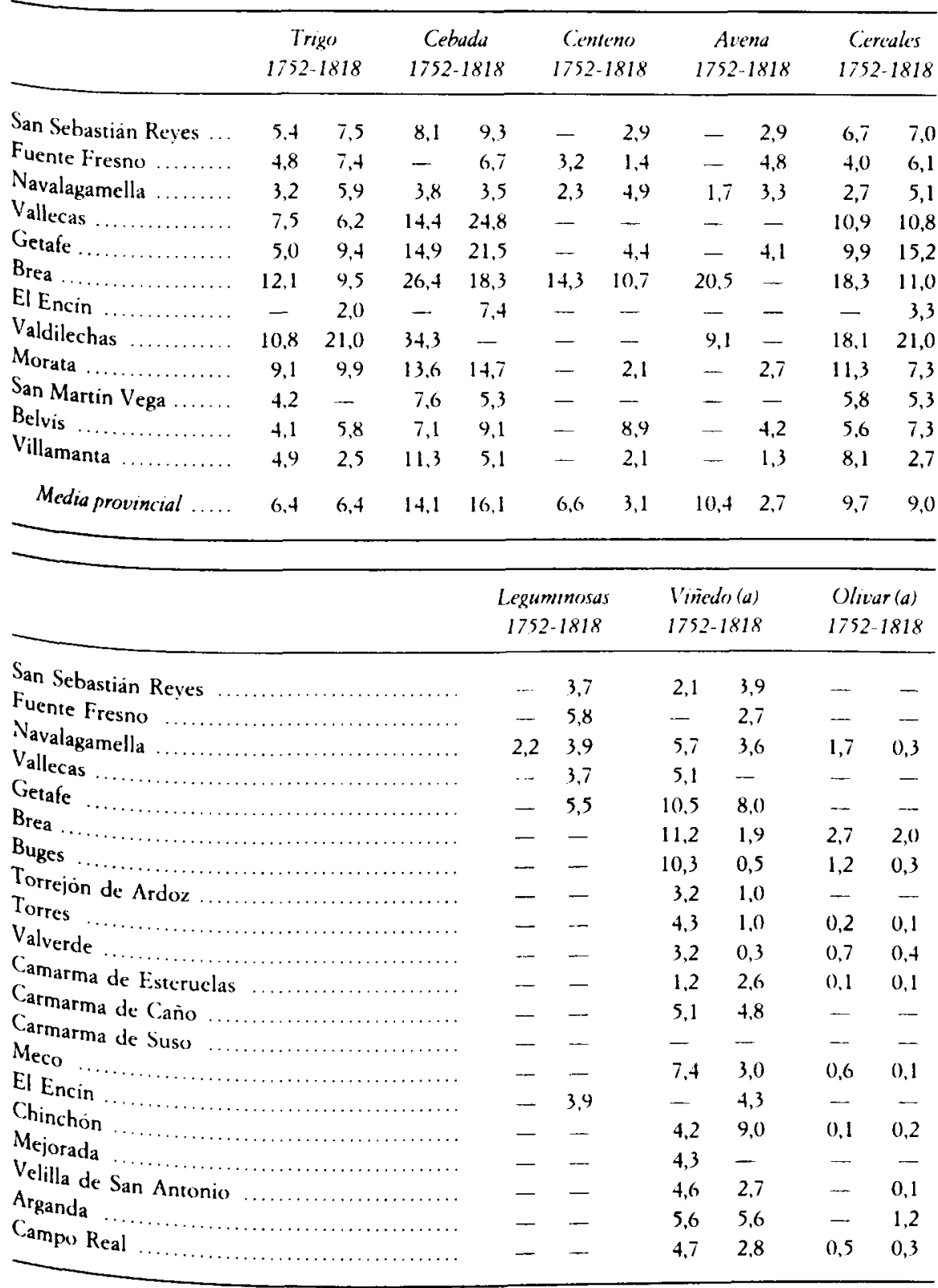




\section{CUADRO A.6 (Continuación)}

Madrid 1752-1818. Productividad superficies sembradas (Qm/ha)

\begin{tabular}{|c|c|c|c|c|c|c|}
\hline \multirow[b]{2}{*}{ Valdilechas } & \multicolumn{2}{|c|}{$\begin{array}{c}\text { Leguminosas } \\
1752-1818\end{array}$} & \multicolumn{2}{|c|}{$\begin{array}{l}\text { Vinedo (a) } \\
1752-1818\end{array}$} & \multicolumn{2}{|c|}{$\begin{array}{c}\text { Olivar (a) } \\
1752-1818\end{array}$} \\
\hline & - & - & 9,5 & 5.8 & 1,1 & 0,6 \\
\hline Orusco & - & - & 10,8 & 4,0 & 1,6 & - \\
\hline Carahaña & - & - & 12,1 & 16,2 & 1,8 & 2,2 \\
\hline Driebes $\ldots \ldots \ldots \ldots \ldots$ & - & - & 6,6 & 5,6 & 2,2 & 一 \\
\hline 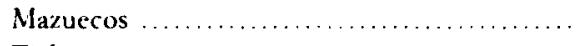 & - & - & 10.8 & 4,8 & 1,2 & 2,8 \\
\hline Tielmes $\ldots \ldots \ldots \ldots \ldots$ & - & - & 10,5 & 10.9 & 1.6 & 1,2 \\
\hline Perale's de Tajuña & - & -- & 13,6 & 6,8 & 0,3 & - \\
\hline Morata ............ & - & 6,8 & 18,0 & 12,0 & 0.5 & 0.1 \\
\hline 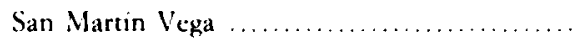 & - & - & 28,0 & 18,8 & - & 一 \\
\hline Valdelaguna $\ldots \ldots \ldots \ldots \ldots \ldots \ldots \ldots \ldots \ldots \ldots \ldots \ldots \ldots$ & - & - & 18,8 & 8,1 & 2,9 & 0,4 \\
\hline Daganzo de Arriba & - & - & 3.5 & 2,5 & - & - \\
\hline Daganzo de Abajo & - & - & 2,8 & 1,5 & - & - \\
\hline Hueros $\ldots \ldots \ldots \ldots$ & - & $\therefore$ & 2.6 & - & 0,6 & - \\
\hline Bayona $\ldots \ldots \ldots \ldots$ & - & $\ldots$ & 7,1 & 4,0 & - & - \\
\hline 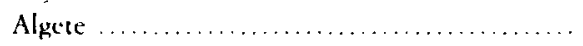 & & - & 1.9 & 3,2 & 0,7 & 54,8 \\
\hline Fresno de Torote $\ldots \ldots \ldots \ldots \ldots \ldots \ldots \ldots$ & - & - & - & 3,7 & - & 1.1 \\
\hline Cobeña $\ldots \ldots \ldots \ldots \ldots \ldots \ldots \ldots \ldots \ldots \ldots \ldots \ldots \ldots \ldots \ldots$ & - & $\ldots$ & 3.5 & - & $\cdots$ & - \\
\hline Belvis $\ldots \ldots \ldots \ldots \ldots$ & .. & 5,9 & $\cdots$ & - & -- & - \\
\hline Ajalvir $\ldots \ldots \ldots \ldots$ & - & - & 1,8 & - & 0,1 & 0,1 \\
\hline Belmonte del Tajo. & - & - & 3,8 & 7,1 & 1,1 & 0,1 \\
\hline Paracuellos ........ & - & $\ldots$ & 5.1 & 1,5 & 1,0 & - \\
\hline$\ldots \ldots \ldots \ldots \ldots \ldots$ & - & 2,6 & 2,6 & 2,8 & - & 0,6 \\
\hline Media pronuncial ........ & 2,2 & 3,9 & 5,4 & 6,7 & 0,5 & 0,2 \\
\hline
\end{tabular}

Fientr: AGS, AHN, Catastro del Marques de la Ensenada y AHN, AMG, AMSSR, AMCh, AMAH, ARM, Cuadernos Generales de la Riqueza.

(a) $\mathrm{Hl}$ ha 


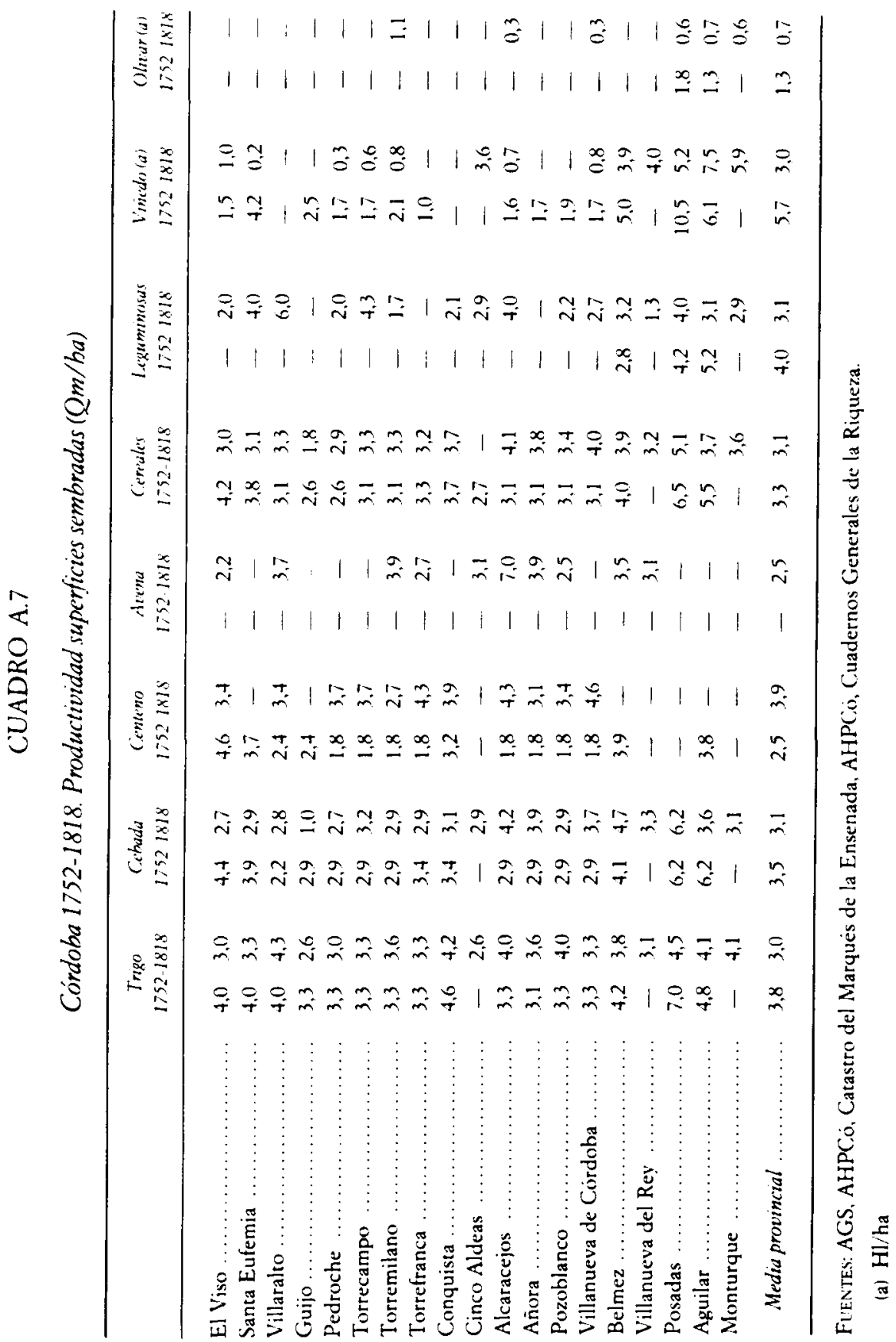




\section{CUADRO A.8}

Alicante 1818. Productividad superficies sembradas $(\mathrm{Qm} / \mathrm{ha})$

\begin{tabular}{|c|c|c|c|c|}
\hline & Trigo & Cébada & Maiz & Cereales \\
\hline Denia & $3 . \overline{7}$ & - & - & 3,7 \\
\hline 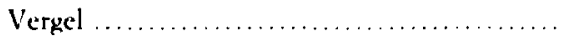 & 2.5 & 2.7 & 2.9 & 2,7 \\
\hline 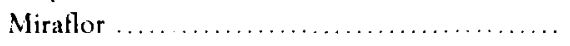 & 6,0 & -- & 2,2 & 4.1 \\
\hline Sedla & - & 3,0 &,+ 2 & 3,6 \\
\hline Beniarbeig & 1,5 & - & - & 1.5 \\
\hline \multirow[t]{2}{*}{ Medra provincial $\ldots \ldots \ldots \ldots \ldots \ldots \ldots \ldots \ldots$} & 3,4 & 2,8 & 3,1 & 3,1 \\
\hline & \multicolumn{2}{|c|}{ Leguminosas } & Vincedo (a) & teas pasas \\
\hline Denia ... & \multicolumn{2}{|c|}{2,2} & 9,1 & 9,4 \\
\hline Vergel .............. & \multicolumn{2}{|c|}{3,0} & 12,3 & 2.1 \\
\hline Miraflor & \multicolumn{2}{|c|}{9,5} & 12,5 & 2,3 \\
\hline Mirarrosa ..... & \multicolumn{2}{|c|}{-} & 9.5 & 2,0 \\
\hline Sedla $\ldots \ldots \ldots \ldots \ldots \ldots \ldots \ldots \ldots \ldots \ldots \ldots \ldots \ldots \ldots \ldots \ldots \ldots \ldots$ & \multicolumn{2}{|c|}{-} & 8,9 & - \\
\hline Rafol $\ldots \ldots \ldots \ldots \ldots \ldots \ldots \ldots \ldots \ldots \ldots \ldots \ldots \ldots \ldots \ldots \ldots \ldots$ & \multicolumn{2}{|l|}{$\ldots$} & - & - \\
\hline Beniarbeig ...... & \multicolumn{2}{|c|}{1,6} & - & - \\
\hline Media provincial & \multicolumn{2}{|c|}{3,6} & 10,4 & 3.9 \\
\hline
\end{tabular}

Fulixr: AMD, Cuadernos Generales de la Riqueza.

(a) $\mathrm{Hl} / \mathrm{ha}$ 


\section{CUADRO A.9}

Segovia 1752-1818. Productividad superficies sembradas ( $(\mathrm{m} / \mathrm{ba})$

\begin{tabular}{|c|c|c|c|c|c|c|c|c|c|c|}
\hline \multirow[b]{2}{*}{ Segovia } & \multicolumn{2}{|c|}{$\begin{array}{c}\text { Trigo } \\
1752-1818 \\
\end{array}$} & \multicolumn{2}{|c|}{$\begin{array}{c}\text { Cethada } \\
1552-1818\end{array}$} & \multicolumn{2}{|c|}{$\begin{array}{c}\text { Comene } \\
1752-1818 \\
\end{array}$} & \multicolumn{2}{|c|}{$\begin{array}{c}\text { Atena } \\
1752 \text { 1818 } \\
\end{array}$} & \multicolumn{2}{|c|}{$\begin{array}{c}\text { Circules } \\
1752-1818\end{array}$} \\
\hline & 4.2 & 6,7 & 12,1 & 9,5 & 2,1 & 3,1 & - & - & 6.1 & 6,9 \\
\hline Fuentepelayo . ................ & 4,8 & 5.1 & 11,1 & 10,7 & 4.2 & 1.7 & - & - & 6,8 & 5,8 \\
\hline San Salvador ..... & - & 5,2 & $\ldots-$ & - & - & 1.7 & - & - & - & 5,2 \\
\hline Reojada. & - & 5.7 & - & $10, \overline{7}$ & - & 1,6 & - & - & - & 6.0 \\
\hline Carra Segovia. & - & 4,9 & $\ldots$ & 10,7 & - & 1,7 & - & - & - & 5,7 \\
\hline Carra $S^{-}$Juan & - & 5,2 & - & $\ldots$ & - & 1,6 & - & - & - & 5,2 \\
\hline Castroserna Arriba & 4,8 & 5.4 & 5,0 & 6,8 & 2,6 & 2,7 & 1.7 & 2,6 & 3,5 & 5,0 \\
\hline Encinas ........... & 5.7 & 7,9 & 10.1 & 7,8 & 4,0 & 5.6 & 2,2 & 3,1 & 5,5 & 7,7 \\
\hline Santa Maria del Cerro & - & 4.7 & - & - & - & 2,8 & - & - & - & 3,9 \\
\hline Ventosilla ............ & 4,3 & 4,7 & 4,6 & - & 2,6 & 3,5 & - & - & 3,8 & 4,1 \\
\hline Pradenilla & 2,4 & 6,2 & 3.2 & 5.7 & 1,3 & 3.5 & $\ldots$ & - & 2,3 & 4,9 \\
\hline Valleruela. & 5.1 & 6.1 & 7,3 & 7,4 & 3,0 & 4.8 & 1,9 & 5,2 & 4,3 & 5.9 \\
\hline Tabladillo. & +1.3 & 4.3 & 6,5 & 5.9 & 2,5 & 3.8 & 2,5 & - & 3,9 & 4,3 \\
\hline Cabezuela. & 6.2 & 8,6 & 7.3 & 14,4 & 2,0 & 3.2 & $\ldots$ & - & 5,1 & 7,8 \\
\hline Remondo. & 6,4 & 3,0 & 14,7 & 8,7 & 2,8 & 0.7 & - & - & 7,9 & 2,4 \\
\hline Coca ..... & 7,5 & 3.6 & 12.4 & 10.5 & 2,8 & 0.9 & - & - & 7,5 & 2,5 \\
\hline Ciruelos. & 6.5 & 4,3 & 15,2 & 11,9 & 2,8 & 0,8 & - & - & 8.1 & 5,5 \\
\hline Media provincial & 5.3 & 6,0 & 8.6 & 9,0 & 2,6 & 2,6 & 2,0 & 3,5 & 5,1 & 5,4 \\
\hline
\end{tabular}

\begin{tabular}{|c|c|c|c|c|}
\hline \multirow[b]{2}{*}{ Segovia } & \multicolumn{2}{|c|}{$\begin{array}{l}\text { Letguminosus } \\
1752-1818\end{array}$} & \multicolumn{2}{|c|}{$\begin{array}{l}\text { Vinedo (a) } \\
1752-1818\end{array}$} \\
\hline & 4,7 & 4,1 & - & - \\
\hline Fuentepelayo & 5,6 & 3,0 & 2.3 & 1,1 \\
\hline San Salvador. & - & 1,8 & - & 2,1 \\
\hline Reojada ...... & - & 3.7 & - & 2,1 \\
\hline Carra Segovia & - & 3,6 & - & 1,8 \\
\hline Carra S. Juan & - & 3,7 & - & 2,0 \\
\hline Encinas ...... & - & 5,9 & - & - \\
\hline Santa Maria del Cerro. & -- & 1.6 & - & - \\
\hline$\ldots \ldots \ldots \ldots \ldots \ldots \ldots \ldots, \ldots$ & - & 2,6 & - & - \\
\hline$\ldots \ldots \ldots \ldots \ldots \ldots \ldots \ldots \ldots \ldots \ldots, \ldots$ & - & 3,8 & - & - \\
\hline$\ldots \ldots \ldots \ldots \ldots \ldots \ldots$ & - & 4,3 & 2,4 & 2,9 \\
\hline$\ldots \ldots \ldots \ldots \ldots \ldots \ldots \ldots \ldots \ldots$, & 4,5 & 8,0 & 3,2 & - \\
\hline 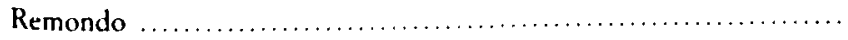 & 5.5 & 3,6 & 2,8 & - \\
\hline$\ldots \ldots \ldots \ldots \ldots \ldots \ldots \ldots$ & 4,7 & 5.0 & 3.9 & 0,7 \\
\hline Ciruelos. & 4,7 & 4,0 & 4,5 & 1,5 \\
\hline Media provincial & 4,9 & 5,2 & 3,1 & 1,1 \\
\hline
\end{tabular}

Fuentr: AGS, AHPS, Catastro del Marqués de la Ensenada, AHPS, AMSe, Cuadernos Generales de la Riqueza.

(a) $\mathrm{Hl} / \mathrm{ha}$ 


\section{CUADRO A.10}

España (pueblos) 1752-1818. Productividad sistema cereal ( $Q \mathrm{~m} / \mathrm{ha})$

\begin{tabular}{|c|c|c|c|c|c|c|}
\hline & \multicolumn{3}{|c|}{1752} & \multicolumn{3}{|c|}{1818} \\
\hline & $P_{l}$ & $P_{2}$ & $S$ & $P_{1}$ & $P_{2}$ & $S$ \\
\hline Santander & 14,8 & - & - & 18,0 & 18,0 & 1,0 \\
\hline Cueto $\ldots \ldots \ldots \ldots \ldots$ & 14,4 & - & -. & 17,8 & 17,8 & 1,0 \\
\hline Monte ................... & 11.8 & - & - & 18,6 & 18,6 & 1,0 \\
\hline San Román ............ & 13,3 & $\ldots$ & - & 19,0 & 19,0 & 1,0 \\
\hline Peñacastillo ...... & 9,3 & - & - & 17,3 & 17,3 & 1,0 \\
\hline Liencres $\ldots \ldots \ldots \ldots \ldots \ldots \ldots \ldots \ldots \ldots \ldots \ldots \ldots$ & 7,4 & - & - & 12,5 & 12,5 & 1.0 \\
\hline 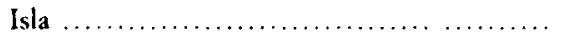 & 9,0 & - & - & 10,0 & 10,0 & 1,0 \\
\hline Villapresente........... & 40,1 & 40,1 & 1,0 & 34,4 & 34,4 & 1,0 \\
\hline Secadura $\ldots \ldots \ldots \ldots$ & 12,0 & 12,0 & 1,0 & 8,0 & 8.0 & 1.0 \\
\hline Villaverde de Trucios & 9,5 & 9.5 & 1,0 & 10,1 & $10, \mathrm{i}$ & 1,0 \\
\hline Puente Viesgo--Aes... & 11,0 & 11,8 & 1,0 & 10,6 & 10,6 & 1,0 \\
\hline 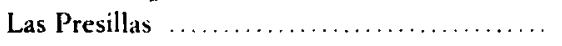 & 5,0 & 5,1 & 1,0 & 15,3 & 15,3 & 1,0 \\
\hline 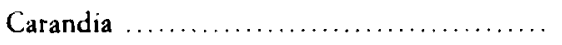 & 6,8 & 6,8 & 1,0 & 12,1 & 12,1 & 1,0 \\
\hline Hijas & 14,9 & - & - & 13,2 & 13,2 & 1.0 \\
\hline Vargas . & 9,8 & - & - & 9,9 & 9,9 & 1,0 \\
\hline Villasevil $\ldots \ldots \ldots \ldots \ldots \ldots \ldots \ldots \ldots \ldots \ldots \ldots \ldots \ldots \ldots \ldots$ & 11,6 & 11.6 & 1,0 & 6,8 & 6,8 & 1,0 \\
\hline 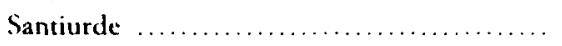 & 23.2 & 23,2 & 1,0 & 24,8 & 24,8 & 1,0 \\
\hline 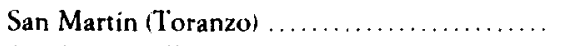 & 18,7 & 18,7 & 1,0 & 6,5 & 6,5 & 1,0 \\
\hline Pando y Penilla $\ldots \ldots \ldots \ldots \ldots \ldots \ldots \ldots \ldots$ & -- & - & - & 8,0 & 8.0 & 1.0 \\
\hline Acereda $\ldots \ldots \ldots \ldots \ldots \ldots \ldots \ldots \ldots \ldots \ldots \ldots \ldots \ldots \ldots \ldots$ & - & - & - & 7,8 & 7,8 & 1,0 \\
\hline Iruz $\ldots \ldots \ldots \ldots \ldots \ldots \ldots \ldots \ldots \ldots \ldots \ldots \ldots \ldots \ldots \ldots \ldots$ & 12,0 & 12,0 & 1,0 & 7,2 & 7,2 & 1,0 \\
\hline Bárcena & 8,5 & 8.5 & 1,0 & 7,4 & 7,4 & 1,0 \\
\hline Vejoris & 9,5 & - & -- & 9,8 & 9,8 & 1,0 \\
\hline 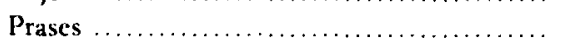 & 10,7 & 10,7 & 1,0 & 6,8 & 6,8 & 1,0 \\
\hline Quintana $\ldots \ldots \ldots \ldots \ldots \ldots \ldots \ldots \ldots \ldots \ldots \ldots \ldots \ldots \ldots \ldots$ & 12.3 & 12,3 & 1,0 & 5,4 & 5.4 & 1,0 \\
\hline Esponzues & 11,0 & 11,0 & 1,0 & 16,4 & 16,4 & 1,0 \\
\hline Corvera $\ldots$ & 12,4 & 12.4 & 1,0 & 7,8 & 7,8 & 1,0 \\
\hline San Vicente & 16,8 & 16.8 & 1,0 & 27,3 & 27,3 & 1,0 \\
\hline Ontaneda .. & 10,7 & 10,7 & 1,0 & 15,3 & 15,3 & 1,0 \\
\hline Alceda $\ldots . . . . .$. & 11,8 & 11,8 & 1,0 & 13,8 & 13,8 & 1,0 \\
\hline Castillo Pedroso $\ldots \ldots \ldots \ldots \ldots \ldots \ldots \ldots$ & 16,8 & 16,8 & 1,0 & 8,7 & 8,7 & 1,0 \\
\hline Borleña y Salcedillo $\ldots \ldots \ldots \ldots \ldots \ldots \ldots$ & 15,5 & 15,5 & 1,0 & 6,6 & 6,6 & 1,0 \\
\hline Villegar $\ldots \ldots \ldots \ldots$ & 15,1 & 15,1 & 1,0 & 26,4 & 26.4 & 1,0 \\
\hline Luena $\ldots \ldots \ldots \ldots \ldots \ldots . . .$. & 14,5 & $\ldots$ & $\ldots$ & 10,4 & 10,4 & 1,0 \\
\hline Resconorio $\ldots \ldots \ldots \ldots \ldots \ldots \ldots \ldots \ldots \ldots \ldots \ldots \ldots \ldots$ & 10,0 & 10,0 & 1,0 & 7,3 & 7,3 & 1,0 \\
\hline Entrambasmestas & 10,5 & 10,5 & 1,0 & 44,5 & 44,5 & 1,0 \\
\hline Cañedo $\ldots \ldots \ldots \ldots \ldots \ldots \ldots \ldots \ldots \ldots \ldots \ldots \ldots \ldots \ldots \ldots \ldots \ldots \ldots$ & 4,6 & 4,6 & 1,0 & 15,1 & 15,1 & 1,0 \\
\hline Santayana $\ldots \ldots \ldots \ldots \ldots \ldots \ldots \ldots \ldots \ldots \ldots \ldots \ldots \ldots \ldots$ & 6.9 & 6,9 & 1,0 & 8,3 & 8,3 & 1,0 \\
\hline
\end{tabular}




\section{CUADRO A.10 (Continuación)}

España (pueblos) 1752-1818. Productividad sistema cereal (Qm/ha)

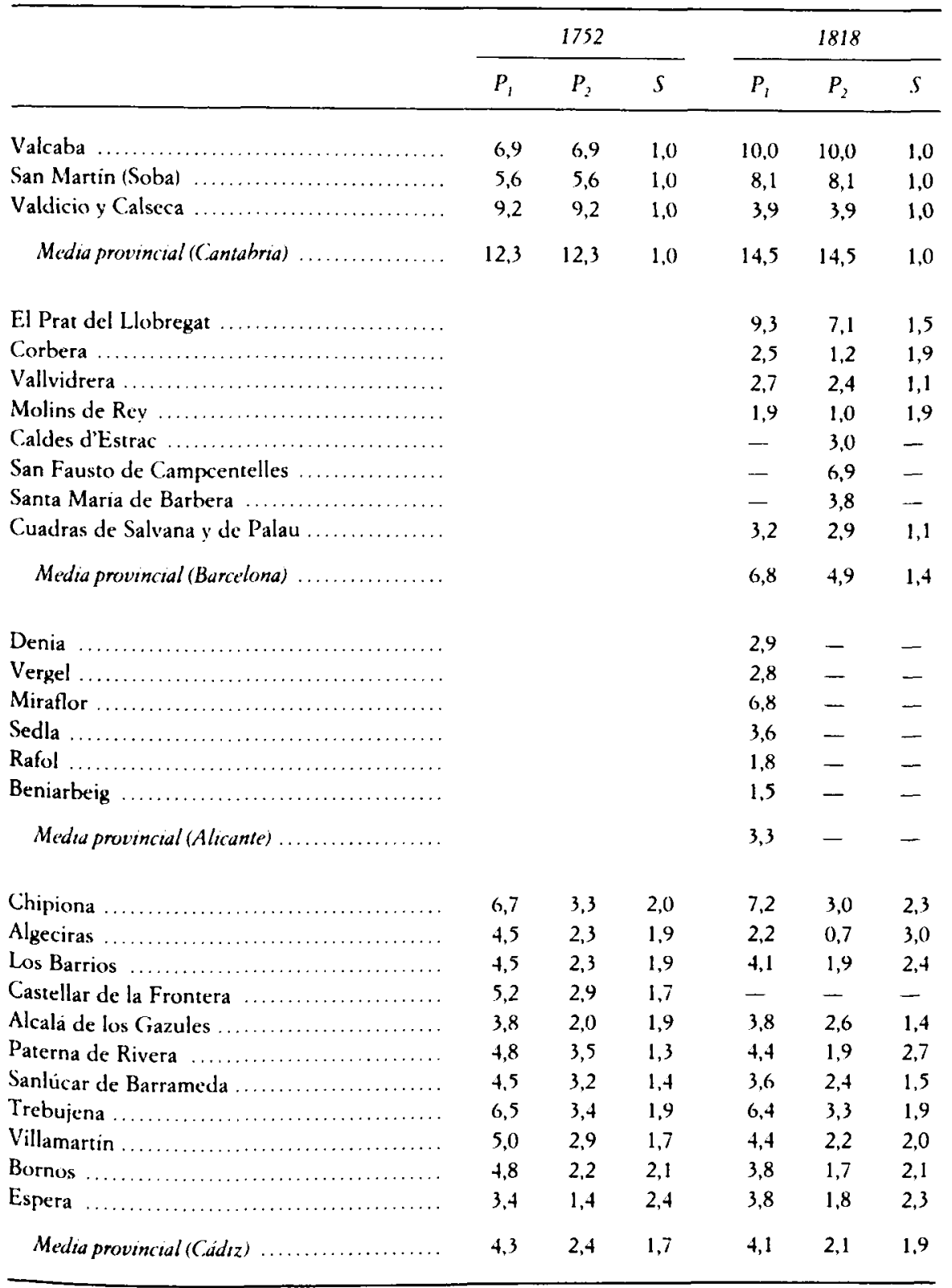




\section{CUADRO A.10 (Continuación)}

España (pueblos) 1752-1818. Productividad sistema cereal $(Q \mathrm{~m} / \mathrm{ba})$

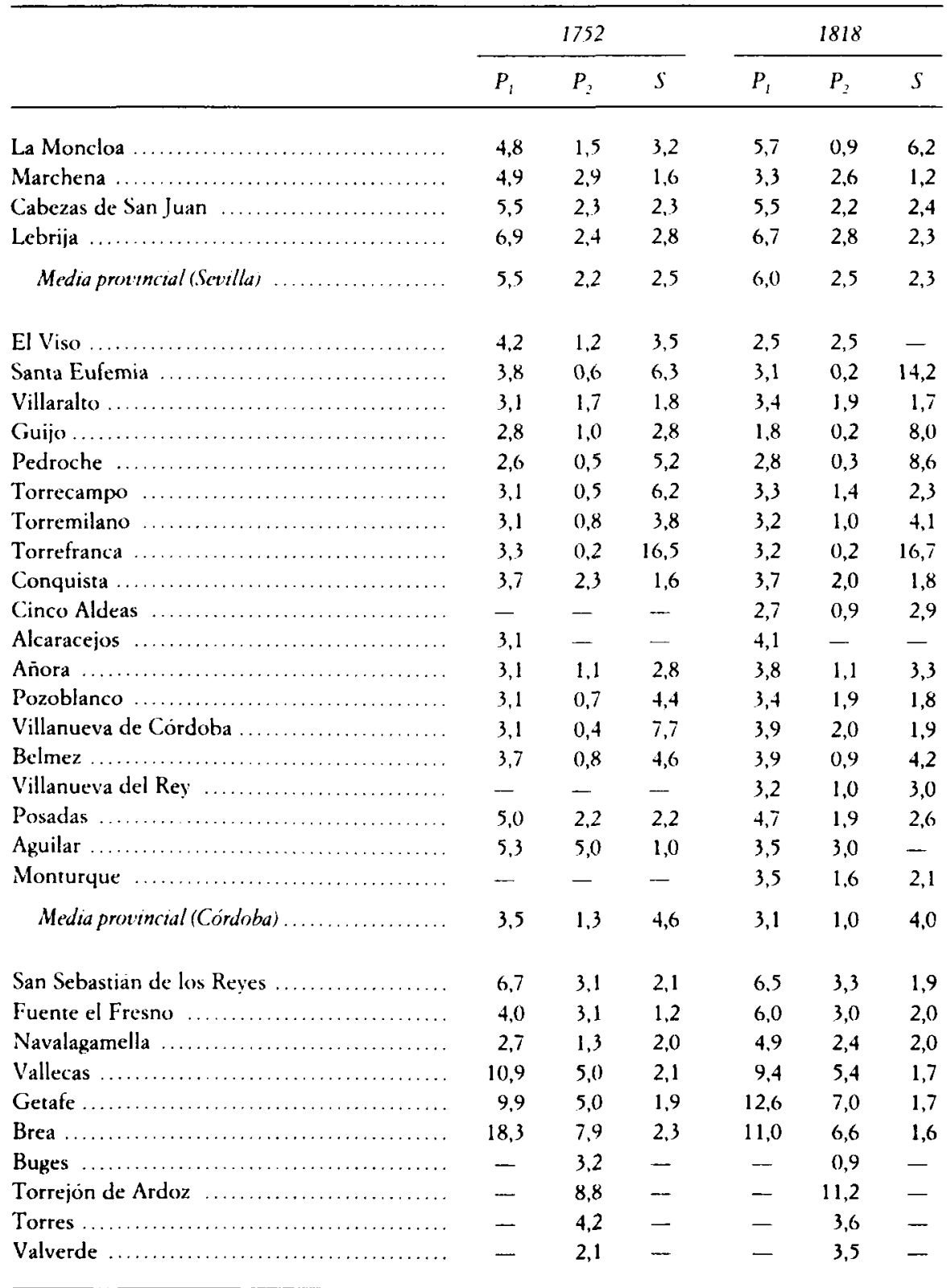




\section{CUADRO A.10 (Continuación)}

España (pueblos) 1752-1818. Productividad sistema cereal ( $(\mathrm{m} / \mathrm{ha})$

\begin{tabular}{|c|c|c|c|c|c|c|}
\hline & \multicolumn{3}{|c|}{1752} & \multicolumn{3}{|c|}{1818} \\
\hline & $P_{1}$ & $P_{2}$ & $s$ & $P_{l}$ & $P_{2}$ & $s$ \\
\hline Camarma de Esteruelas & - & 3,3 & - & - & 2,0 & - \\
\hline Carmarma de Caño .... & - & 3,5 & -- & -- & 3,8 & - \\
\hline Carmarma de Suso & - & $\ldots$ & - & - & 2,3 & - \\
\hline 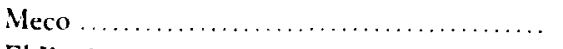 & - & 4,2 & - & - & 4,1 & -- \\
\hline El Encín $\ldots \ldots \ldots \ldots \ldots \ldots \ldots \ldots \ldots \ldots \ldots \ldots \ldots \ldots \ldots \ldots$ & - & - & - & 3,3 & $\cdots$ & - \\
\hline Chinchón $\ldots \ldots \ldots \ldots \ldots \ldots \ldots \ldots \ldots \ldots . \ldots \ldots$ & - & 3,3 & - & - & 2,9 & - \\
\hline Mejorada $\ldots \ldots \ldots \ldots$ & - & 4,9 & - & - & 1,2 & - \\
\hline Velilla de $S_{a n}$ Antonio $\ldots \ldots \ldots \ldots \ldots \ldots$ & - & 3,4 & - & - & 1,7 & - \\
\hline Arganda $\ldots \ldots \ldots \ldots \ldots \ldots \ldots \ldots \ldots \ldots \ldots$ & - & 2,6 & $\ldots$ & - & 3,5 & - \\
\hline Campo Real & $\ldots$ & 2,0 & - & - & 2,0 & - \\
\hline Valdilechas ...... & 18,1 & 5,1 & 3.5 & - & 11,1 & - \\
\hline 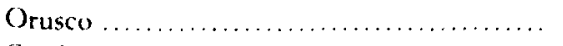 & - & 5,8 & - & - & 5,0 & - \\
\hline Carabaña $\ldots \ldots \ldots \ldots \ldots \ldots \ldots \ldots \ldots \ldots \ldots \ldots \ldots \ldots \ldots$ & - & 5,5 & - & - & 19,7 & - \\
\hline Driebes $\ldots \ldots \ldots \ldots \ldots \ldots \ldots \ldots \ldots \ldots \ldots \ldots \ldots \ldots \ldots \ldots$ & - & 8,7 & - & - & 5,9 & - \\
\hline Mazuecos $\ldots \ldots \ldots \ldots$ & $\cdots$ & 7,3 & - & - & 3,7 & - \\
\hline Tielmes $\ldots . . . \ldots \ldots$ & - & 12,9 & - & - & 23,8 & - \\
\hline Perales de Tajuña $\ldots . . .$. & - & 3.0 & - & - & 3,2 & - \\
\hline Morata $\ldots \ldots \ldots \ldots \ldots$ & 11,3 & 5,9 & 1,9 & - & 6,9 & - \\
\hline Valdelaguna..... . & - & 5,3 & - & - & 2,7 & - \\
\hline Daganzo de Arriba $. . . \ldots \ldots . .$. & - & 4.2 & - & - & 2,6 & - \\
\hline Daganzo de Abajo ....................... & --- & 2,9 & - & - & 2,5 & - \\
\hline 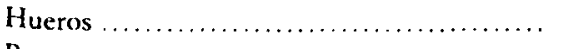 & - & 3,4 & - & - & 2,8 & - \\
\hline Bayona $\ldots \ldots \ldots \ldots \ldots$ & - & 9,9 & - & - & 2,7 & - \\
\hline Algete $\ldots \ldots \ldots \ldots \ldots$ & - & 3,5 & - & - & 3,1 & - \\
\hline Fresno de Torote $\ldots \ldots \ldots \ldots \ldots \ldots \ldots \ldots$ & - & 3,7 & - & - & 1,1 & - \\
\hline 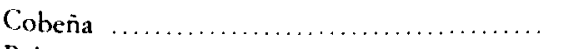 & - & 4,9 & - & - & 3.4 & - \\
\hline Belvis $\ldots \ldots \ldots \ldots \ldots \ldots$ & 5,6 & 2,8 & 2,0 & 7,2 & 3,6 & 1,9 \\
\hline Ajalvir ................. & - & 2,3 & - & - & 3,0 & - \\
\hline Paracuellos & - & 3,1 & - & - & 3,0 & - \\
\hline Belmonte del Tajo ....................... & - & 2,9 & - & - & 3,0 & - \\
\hline Villamanta $\ldots \ldots \ldots \ldots \ldots \ldots \ldots \ldots \ldots \ldots \ldots$ & 8,1 & 2.5 & 3,2 & 2,7 & 1,0 & 2,7 \\
\hline Media provincial (Madrid) & 7,8 & 3,8 & 2,0 & 8,0 & 4,1 & 1,9 \\
\hline Segovia ....... & 5,7 & 2,8 & 2,0 & 6,8 & 3,5 & 1,9 \\
\hline Fuentepelayo .. & 6.5 & 3,3 & 1,9 & 5,8 & 3,7 & 1,5 \\
\hline San Salvador.. & - & - & - & 5,2 & - & - \\
\hline Reojada ....... & - & - & - & 5,4 & - & - \\
\hline Carra Segovia & - & - & - & 5,2 & - & - \\
\hline
\end{tabular}




\section{CUADRO A.10 (Continuación)}

España (pueblos) 1752-1818. Productividad sistema cereal ( $Q m / b a)$

\begin{tabular}{|c|c|c|c|c|c|c|}
\hline & \multicolumn{3}{|c|}{1752} & \multicolumn{3}{|c|}{1818} \\
\hline & $P_{1}$ & $P_{z}$ & $s$ & $P_{i}$ & $P_{z}$ & $s$ \\
\hline Carra S. Juan & - & - & - & 4,4 & - & - \\
\hline Encinas $\ldots \ldots \ldots \ldots$ & 5,5 & 2,9 & 1,8 & 7.7 & 4,0 & 1,8 \\
\hline Santa Maria del Cerro. & - & - & $\ldots$ & 3.9 & 1,7 & 2,2 \\
\hline 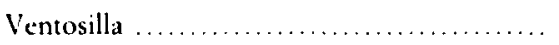 & 3,8 & 1,5 & 2,5 & 4,1 & 1,9 & 2,1 \\
\hline Pradenilla $\ldots . . . \ldots \ldots \ldots \ldots$ & 2,3 & 0.8 & 2.8 & 4,9 & 2.5 & 1,9 \\
\hline Castroserna de Arriba. & 3,5 & 1,5 & 2,3 & 5.1 & 2.1 & 2,3 \\
\hline Valleruela de Sepulveda. & 4,3 & 1,8 & 2,3 & 5,9 & 3,1 & 1,8 \\
\hline Tabladillo ............... & 3.9 & 1,5 & 2.6 & 4,3 & 2,5 & 1.6 \\
\hline Cabezuela ..... & 5,0 & 2,1 & 2,3 & 7,8 & 3,9 & 1,9 \\
\hline Remondo ................. & 6.7 & 3.2 & 2,0 & 2.5 & 1.1 & 2,2 \\
\hline Coca & 6,8 & 3,2 & 2,1 & 2,7 & 1,2 & 2.1 \\
\hline Ciructos de Coca $\ldots . . . \ldots \ldots \ldots$. & 7,3 & 4,7 & 1,5 & 5.1 & 2.7 & 1,8 \\
\hline Medua provincial (Segoria) ..................... & 5.0 & 2.6 & 1,9 & 5,4 & 2,7 & 1,9 \\
\hline Poza de la Sal (Burgos) ..... & 3,0 & - & - & 5,6 & 3.4 & 1,6 \\
\hline Pinilla de Toro (Zamora) ...................... & 5.6 & 3.5 & 1,6 & 6,3 & 3.4 & 1,8 \\
\hline Talavera Reina (Toledol ..................... & 4,3 & 3,1 & 1,3 & 7,1 & 6,8 & 1,0 \\
\hline Castellón Plana (Castellón) .................... & - & - & - & 2.7 & 2.7 & 1,0 \\
\hline Linares (Jaen) . .............................. & 5.8 & 2,3 & 2,5 & 5.5 & - & - \\
\hline
\end{tabular}

Funtr: AGS, AHN, AHPC, AHPS, AHPCo, AMM, Catastro del Marques de la Ensenada y AHPC, ADS, AMS, BPS, AGDB, AMD, AHPCa, AHPCa, AME, AMM, AHPCó, AHN, ARM, AMSSR, AMG, AMAH, AMCh, AHPS, AMPS, AMTT, AMTR, AMC. AHPJ, Cuadernos Generales de la Riqueza.

$P_{1}$ - Productividad superficies sembradas.

$P_{2}$ - Productividad superficies cultivadas.

$S$ - Superficies cultivadas superficies sembradas.

\section{BIBLIOGRAFIA}

Anónimo (1948): «Las publicaciones de la "Junta Consultiva Agronomica" ", Boletín bibliográfico agrícola, núm. 6, pp. 225-229.

AbELA Y SAIN dE ANDivo, E. (1876): Memoria sobre el estado de la agricultura en la provincia de Madrid y mejoras convenientes para su desarrollo. Madrid.

Amalric, J. P., y Bremont, F. (1975): «Evolución de las estructuras agrarias en la Casti- 
lla Moderna: el ejemplo de la Bureba», Metodologia de la Historia Moderna. Economía y demografía, pp. 225.238, Santiago de Compostela.

Avalkic, J. P. (1990): Peuplement, paysage, production en Vieille Castille au XVIII siecle, 2 vols. Tesis Doctoral inédita. Ioulouse.

ANEs Alvakl. G. G. (1974): Las crisis agrarias en la España Moderna, Madrid.

Baiko(11, P. (1989): "Les trois révolutions agricoles du monde développé: rendements et productivite de 1800 a 1985m, Annales Economies Societés Civilisations, num. 2, pp. 317.353.

BarbieRo, T. P. (1988): «A reassessment of agricultural production in Italy 1861-1914: the case of Lombardy». The lournal of European Economic History, num. 1, pp. 103. 116.

Bi:knat. Ronrlielez, A. M. (1991): «Resistencias al cambio económico desde el sector agricola (1880-1931);; GARcL Del.ciado, J. L. led): España entre dos siglos (1875-1931). Continuidad y cambio, pp. 141-156, Madrid.

Bremont, F. (1979): "Comptes d'exploitation et histoire economique: l'exemple de la "granja" de Quintanajuar», Melanges de la Casa de Velazquez, tomo XIV, pp. 285-413.

- (1985): «Une exploitation en faire-valoir direct: la granje d'Hocina (1685-1707)m; Ponsot, P., y Amalric, I. P. (eds.): Lexploitation des grands domaines dans lEspagne d'Ancien Regime, pp. 133-160, Paris.

Camariro But.on, C. (1984): "La producción agraria en el siglo xvill. El Catastro de Ensenada y las certificaciones de diezmos como fuentes para el estudio de la producción y de los rendimientos agricolas. Análisis de un caso real: Gumiel de Hizán, 1748-1753m, Estudios Geográficos, núm. 174, pp. 81-107.

Canpieit., B. M. S., y Ov':RToN, M. (eds.) (1991): Land, labour and livestosk: bistorical studies in european agricultural productivity, Manchester.

FLoRES IE LEMt'S, A. (1976): «Algunos datos estadisticos sobre el estado actual de la economia española», Hacienda Pública Española, núm. 42.43, pp. 421.465.

Fionistan Imizi:oz, A. (1982): La Merindad de Estella en la edad moderna: los bombres y la tierra, Pamplona.

Garcta SAN/, A. (1985): «La granje de Nieva du Monastere du Parral (Segovie) entre 1739 et 1808»; Ponsot, P., y Allalkic, J. P. (eds.): Lexploitation des grands domaines dans l'Espagnc d'Ancien Regime, pp. 193.221, Paris.

- (1986): Desarrollo y crisis del Antiguo Régimen en Castilla la Vieja. Economia y sociedad en tierras de Segovia de 1500 a 1814 . Madrid.

Garkabou, R. (1978): «Cultius, collites i rendiments a la Segarra i Alt Anoia: els comptes d'unes finques de Guissona, Sant Marti i Castellfollit de Riubregós (1847. 18691", Estudis d Historia Agraria, num. 1, pp. 241-280.

GARRABOU, R., y SMNL, J. (1985): «La agricultura espanola durante el siglo XIX: ¿inmovilismo o cambio?", en Garkabou, R., y Savz, J. leds.): Historia agraria de la España Contemporánea, vol. II, pp. 7-191, Barcelona.

Grtpo de Estudios de. Historia RuRal (1983): “Notas sobre la producción agraria española, 1891-1931", Revista de Historia Económica, num. 2, p., 185.252.

- (1991): Estadisticas bistóricas de la producción agraria española, 1859-1935, Madrid.

Heltz de. Lemes, A. (1967): Vignobles et vins du Nord-Ouest, 2 tomos, Bordeaux.

JTAN VIDAL, J. (1978): «I'écnicas, rendimientos y productividad agricola en la Mallorca modernas; Axis Alvakez, G., y otros: La conomía agraria en la bistoria de España. Propiedad, explotación, comercialización, renta. pp. 47.56, Madrid. 
Llopts AciLLAN, E. (1980): Las economias monásticas al final del Antiguo Rígimen e'n Extre' madura, Madrid.

Nusez Pestavo, J. R. (1984): La dinámica de la propiedad de la tierra en Icad de los Vinos (1796-1830). Transformaciones sociales y comportamiento económico en la crisis del Antiguo Régimen. La Laguna.

Preklz Gakcla, J. M. (1979): Un modelo de sociedad rural de Antiguo Régimen en la Galicia costera: la Peninsula del Salnés (Jurisdicción de La Lanzada), Santiago.

Poxisor, P. (1982): "Malthus nétait-il pas prophète en Andalousie? Les rendements des cereales en Basse Andalousie, XVII ${ }^{\circ}$ XIX siecles»; (joY, J., y LF. RoY LADURIE, E. (eds.): Prestations paysannes dimes, rente fontière et mouvement de la production agricole a l'́poque préindustrielle, tomo I, pp. 431-446, Paris.

Ponsot, P. (1985) "Les comptes d'une hacienda oleicole andalouse au XVIIIe siegle: La Guijarrosa»; Ponsor, P., y AmAikli, J. P. (dir.): L'exploitation des grands domaines dans l'Espagne d'Ancien Régime, pp. 177.192, Paris.

Prados de la Escosura, L. (1988): De Imperio a Nación. Crecimiento y atraso conómico en España (1780-1930), Madrid.

SANCHLZ-AlboRn(\%, N. (1977): España bace un siglo: una economia dual, Madrid.

Santoveñ Setien, A., y Gutifrezez Brincias, M. A. (1991): "Los cuadernos de la riqueza (1817-1820): una fuente para el estudio de la historia rural española». Revista de Historia Económica, núm. 3, pp. 553-560.

Simpson, J. (1989): «La producción agraria y el consumo español en el siglo xix», Revisla de Historia Económica, num. 2, pp. 364-388.

Tortfilla Casarls, G. (1981): “La economia española, 1830-1900m, en TuNón de Lara, M. (ed.): Historia de España, vol. VIII, pp. 9-167, Madrid.

VAN ZANDEN, J. L. (1991): "The first green revolution: the growth of production and productivity in European agriculture, 1870-1914\%, The Economic Histony Revieu: núm. 2, pp. 215.239.

VIceNs VIVEs, J. (1977): Manual de bistoria económica de España, Barcelona.

VILAR, P. (1967): «L'explotation agricole d'une propieté dans l'horta de Tärrega», Homenaje a Jaime Vicens Vives, tomo II, pp. 761-783, Barcelona. 